MATHEMATICS OF COMPUTATION

Volume 75, Number 256, October 2006, Pages 1675-1696

S $0025-5718(06) 01864-3$

Article electronically published on July 3, 2006

\title{
AN A PRIORI ERROR ANALYSIS \\ FOR THE COUPLING OF LOCAL DISCONTINUOUS GALERKIN AND BOUNDARY ELEMENT METHODS
}

\author{
GABRIEL N. GATICA AND FRANCISCO-JAVIER SAYAS
}

\begin{abstract}
In this paper we analyze the coupling of local discontinuous Galerkin (LDG) and boundary element methods as applied to linear exterior boundary value problems in the plane. As a model problem we consider a Poisson equation in an annular polygonal domain coupled with a Laplace equation in the surrounding unbounded exterior region. The technique resembles the usual coupling of finite elements and boundary elements, but the corresponding analysis becomes quite different. In particular, in order to deal with the weak continuity of the traces at the interface boundary, we need to define a mortar-type auxiliary unknown representing an interior approximation of the normal derivative. We prove the stability of the resulting discrete scheme with respect to a mesh-dependent norm and derive a Strang-type estimate for the associated error. Finally, we apply local and global approximation properties of the subspaces involved to obtain the a priori error estimate in the energy norm.
\end{abstract}

\section{INTRODUCTION}

The local discontinuous Galerkin method is nowadays a very well-established numerical tool to solve a large class of diffusion dominated and purely elliptic equations (see, e.g., 8], 10, 11], and [28]). Moreover, as shown recently in [5] and [6], the applicability of this approach also includes some nonlinear boundary value problems in heat conduction and fluid mechanics. In fact, the results from [28] are extended in [5] to a class of nonlinear diffusion problems with mixed boundary conditions, whereas a new mixed LDG method for certain nonlinear models appearing in quasi-Newtonian Stokes fluids is studied in 6]. We also refer to 22 for related results dealing with the extension of the interior penalty DG method to quasilinear elliptic equations. As already emphasized by many authors, the main advantages of the LDG methods are the high order of approximation provided, the high degree of parallelism involved, and its suitability for $h, p$, and $h p$ refinements. In particular, the latter has motivated the development of associated a posteriori error estimators

Received by the editor January 3, 2005 and, in revised form, August 31, 2005.

2000 Mathematics Subject Classification. Primary 65N30, 65N38, 65N12, 65 N15.

Key words and phrases. Boundary elements, local discontinuous Galerkin, coupling, error estimates.

This research was partially supported by CONICYT-Chile through the FONDAP Program in Applied Mathematics, by the Dirección de Investigación of the Universidad de Concepción through the Advanced Research Groups Program, by Spanish FEDER/MCYT Project MTM2004-019051, and by a grant of Programa Europa XXI (Gobierno Aragón + CAI).

(C)2006 American Mathematical Society 
allowing the implementation of adaptive DG and LDG methods (see, e.g., 3], 4, [6], and [29]).

On the other hand, not much has been done in connection with the combination of LDG and other procedures for elliptic boundary value problems. To this respect, we can only refer to [12, [14, 15, [16, 27, and [31, where the coupling of either DG or LDG with continuous finite element methods (FEM) has been analyzed. For instance, a coupled continuous-DG method is studied in [14 for the numerical solution of linear convection-diffusion problems, and the coupling of the LDG method with the Raviart-Thomas mixed-FEM is proposed in [12 for elliptic equations modeling flow problems. Similarly, the coupling of continuous and DG methods is utilized in [16] to develop a new discrete scheme for two-dimensional shallow water equations. Nevertheless, to the authors' knowledge, the coupling of LDG with another widely applicable procedure, such as the boundary element method (BEM), has not been investigated yet. The suitability of BEM for homogeneous linear boundary value problems in bounded and unbounded domains is well known. Furthermore, the coupling of BEM and FEM is already recognized as a very powerful technique for solving a large class of transmission problems in physics and engineering sciences (see, e.g., 77, 18, 19, 21, 23, 25, 26, 24, 30, and the references therein). This procedure combines the advantage of BEM for treating homogeneous domains with that of FEM for dealing with linear and nonlinear materials. However, when the solution in the FEM region is known to be rough, an LDG method is certainly more appropriate for its approximation. In particular, LDG does not require any continuity condition across the interelement boundaries, it is robust with respect to discontinuous coefficients, and it allows the use of different polynomial degress in each element.

According to the above, and motivated by the need of further developments of combined methods, we now propose to apply LDG instead of FEM, thus yielding the coupling of LDG and BEM. It is important to point out in advance that the coupling of BEM with any other DG methods could be obtained with essentially the same approach to be developed here. As the starting point of the analysis for the coupled approach, we concentrate here on linear exterior boundary value problems arising in potential theory. In forthcoming works we will address nonlinear behaviours and other areas of application. In order to describe our present model problem, we first let $\Omega_{0}$ be a simply connected and bounded domain in $\mathbb{R}^{2}$ with polygonal boundary $\Gamma_{0}$. Then, given $f \in L^{2}\left(\mathbb{R}^{2} \backslash \bar{\Omega}_{0}\right)$ with compact support, we consider the exterior Dirichlet problem:

$$
-\Delta u=f \quad \text { in } \quad \mathbb{R}^{2} \backslash \bar{\Omega}_{0}, \quad u=0 \quad \text { on } \quad \Gamma_{0}, \quad u(\mathbf{x})=\mathcal{O}(1) \quad \text { as } \quad|\mathbf{x}| \rightarrow+\infty .
$$

The Dirichlet boundary condition on $\Gamma_{0}$ has been taken homogeneous only for simplicity of the presentation. The analysis in this paper carries over, with minor modifications, to the case $u=g$ on $\Gamma_{0}$, with $g$ sufficiently smooth. We also remark that the behavior of $u$ at infinity can be described, equivalently, by demanding that $u \in W^{1}\left(\mathbb{R}^{2} \backslash \bar{\Omega}_{0}\right)$ (see [17] for a definition of this Beppo-Levi space). Next, let $\Gamma$ be another simple closed polygonal curve such that the support of $f$ falls inside the annular domain $\Omega$ determined by $\Gamma_{0}$ and $\Gamma$. We further assume that this support does not intersect $\Gamma$. Then, (1.1) can be split, equivalently, as a Poisson equation in $\Omega$ :

$$
-\Delta u=f \quad \text { in } \Omega, \quad u=0 \text { on } \Gamma_{0},
$$


and Laplace equation in the unbounded region $\Omega_{e}:=\mathbb{R}^{2} \backslash\left(\bar{\Omega}_{0} \cup \bar{\Omega}\right)$ :

$$
-\Delta u=0 \quad \text { in } \quad \Omega_{e}, \quad u(\mathbf{x})=\mathcal{O}(1) \text { as }|\mathbf{x}| \rightarrow+\infty,
$$

coupled with the transmission conditions:

$$
\lim _{\substack{\mathbf{x} \rightarrow \mathbf{x}_{0} \\ \mathbf{x} \in \Omega}} u(\mathbf{x})=\lim _{\substack{\mathbf{x} \rightarrow \mathbf{x}_{0} \\ \mathbf{x} \in \Omega_{e}}} u(\mathbf{x}) \text { and } \lim _{\substack{\mathbf{x} \rightarrow \mathbf{x}_{0} \\ \mathbf{x} \in \Omega}} \nabla u(\mathbf{x}) \cdot \nu\left(\mathbf{x}_{0}\right)=\lim _{\substack{\mathbf{x} \rightarrow \mathbf{x}_{0} \\ \mathbf{x} \in \Omega_{e}}} \nabla u(\mathbf{x}) \cdot \nu\left(\mathbf{x}_{0}\right)
$$

for almost all $\mathbf{x}_{0} \in \Gamma$, where $\nu\left(\mathbf{x}_{0}\right)$ denotes the unit outward normal to $\mathbf{x}_{0}$.

The main purpose of this work is to numerically solve (1.1) by means of the coupled LDG-BEM approach, which basically consists of applying LDG to (1.2) and BEM to (1.3). The rest of the paper is organized as follows. In Section 2 we derive the resulting discrete scheme. This includes the boundary integral equation formulation for the exterior problem (1.3), the LDG setting of the interior problem (1.2), the introduction of the auxiliary mortar-type unknown, and then the coupled LDG-BEM scheme. In Section 3 we define appropriate mesh-dependent norms and prove the unique solvability and stability of the coupled method. Finally, in Section 4 we deduce a Strang-type estimate and prove the corresponding a priori error estimate, which is shown to be optimal with respect to all the meshsizes involved, except for the one associated to the mortar-type unknown. Nevertheless, we also show that the regularity of the solution in the exterior region allows us to circumvent this lack of optimality.

Throughout this paper, $c$ and $C$, with or without subscripts, bars, tildes or hats, denote positive constants, independent of the parameters and functions involved, which may take different values at different occurrences. In addition, given any linear space $V$, the corresponding vector-valued space $V \times V$, endowed with the product norm, will be denoted in boldface $\mathbf{V}$. If $\mathcal{O}$ is an open set, its closure, or a polygonal curve, and $s \in \mathbb{R}$, then $|\cdot|_{s, \mathcal{O}}$ and $\|\cdot\|_{s, \mathcal{O}}$ denote the seminorm and norm in the Sobolev space $H^{s}(\mathcal{O})$. In particular, the norms of $H^{s}(\Gamma)$ are denoted $\|\cdot\|_{s, \Gamma}$. Also, $\langle\cdot, \cdot\rangle$ denotes both the $L^{2}(\Gamma)$ inner product and its extension to the duality pairing of $H^{-s}(\Gamma) \times H^{s}(\Gamma)$.

\section{The COUPLED LDG-BEM APPROACH}

We first follow [10] (see also [5, 8], and [28) and introduce the gradient $\sigma:=$ $\nabla u$ in $\Omega$ as an additional unknown. Hereafter, $u$ is the exact solution of (1.2)-(1.3). Also, we define the following auxiliary quantities that will later act as unknowns:

$$
\lambda\left(\mathbf{x}_{0}\right):=\lim _{\substack{\mathbf{x} \rightarrow \mathbf{x}_{0} \\ \mathbf{x} \in \Omega}} \nabla u(\mathbf{x}) \cdot \nu\left(\mathbf{x}_{0}\right), \quad \gamma\left(\mathbf{x}_{0}\right):=\lim _{\substack{\mathbf{x} \rightarrow \mathbf{x}_{0} \\ \mathbf{x} \in \Omega_{e}}} \nabla u(\mathbf{x}) \cdot \nu\left(\mathbf{x}_{0}\right),
$$

and

$$
\varphi\left(\mathbf{x}_{0}\right):=\lim _{\substack{\mathbf{x} \rightarrow \mathbf{x}_{0} \\ \mathbf{x} \in \Omega_{e}}} u(\mathbf{x})-\kappa \quad \text { with } \quad \kappa:=\frac{1}{|\Gamma|} \int_{\Gamma} u,
$$

for almost all $\mathbf{x}_{0} \in \Gamma$. In this way, (1.2) can be reformulated as

$$
\sigma=\nabla u \quad \text { in } \Omega, \quad-\operatorname{div} \sigma=f \quad \text { in } \quad \Omega, \quad u=0 \quad \text { on } \Gamma_{0},
$$

and the transmission conditions (1.4) become

$$
\lim _{\substack{\mathbf{x} \rightarrow \mathbf{x}_{0} \\ \mathbf{x} \in \Omega}} u(\mathbf{x})=\varphi\left(\mathbf{x}_{0}\right)+\kappa \quad \forall(\text { a.e. }) \mathbf{x}_{0} \in \Gamma \quad \text { and } \quad \lambda=\gamma \quad \text { on } \quad \Gamma .
$$


2.1. The boundary integral formulation in the exterior domain. We begin with Green's representation formula for $u$ in $\Omega_{e}$, that is,

$$
u(\mathbf{x}):=\int_{\Gamma} \partial_{\nu(\mathbf{y})} E(\mathbf{x}, \mathbf{y}) \varphi(\mathbf{y}) \mathrm{d}_{s} \mathbf{y}-\int_{\Gamma} E(\mathbf{x}, \mathbf{y}) \gamma(\mathbf{y}) \mathrm{d}_{s} \mathbf{y} \quad \forall \mathbf{x} \in \Omega_{e},
$$

where $E(\mathbf{x}, \mathbf{y}):=-\frac{1}{2 \pi} \log |\mathbf{x}-\mathbf{y}|$ is the fundamental solution of the two-dimensional Laplacian.

It follows from (2.3) and the jump conditions of the layer potentials that

$$
\begin{array}{ccccc}
\mathcal{W} \varphi-\left(\frac{1}{2} \mathcal{I}-\mathcal{K}^{\prime}\right) \gamma & =-\gamma & \text { on } \Gamma, \\
\left(\frac{1}{2} \mathcal{I}-\mathcal{K}\right) \varphi+\mathcal{V}_{\gamma}+\kappa & =0 & \text { on } \Gamma,
\end{array}
$$

where $\mathcal{V}, \mathcal{K}, \mathcal{K}^{\prime}$, and $\mathcal{W}$ denote the boundary integral operators associated to the single, double, adjoint of the double, and hypersingular layer potentials, respectively. We recall from 13 that their main mapping properties are given by $\mathcal{V}: H^{-1 / 2}(\Gamma) \rightarrow H^{1 / 2}(\Gamma), \mathcal{K}: H^{1 / 2}(\Gamma) \rightarrow H^{1 / 2}(\Gamma), \mathcal{K}^{\prime}: H^{-1 / 2}(\Gamma) \rightarrow H^{-1 / 2}(\Gamma)$, and $\mathcal{W}: H^{1 / 2}(\Gamma) \rightarrow H^{-1 / 2}(\Gamma)$, and that they are defined as follows:

$$
\begin{gathered}
\mathcal{V} \mu(\mathbf{x}):=\int_{\Gamma} E(\mathbf{x}, \mathbf{y}) \mu(\mathbf{y}) \mathrm{d}_{s} \mathbf{y} \quad \forall(\text { a.e. }) \mathbf{x} \in \Gamma, \forall \mu \in H^{-1 / 2}(\Gamma), \\
\mathcal{K} \psi(\mathbf{x}):=\int_{\Gamma} \partial_{\nu(\mathbf{y})} E(\mathbf{x}, \mathbf{y}) \psi(\mathbf{y}) \mathrm{d}_{s} \mathbf{y} \quad \forall(\text { a.e. }) \mathbf{x} \in \Gamma, \forall \psi \in H^{1 / 2}(\Gamma), \\
\mathcal{K}^{\prime} \mu(\mathbf{x}):=\int_{\Gamma} \partial_{\nu(\mathbf{x})} E(\mathbf{x}, \mathbf{y}) \mu(\mathbf{y}) \mathrm{d}_{s} \mathbf{y} \quad \forall(\text { a.e. }) \mathbf{x} \in \Gamma, \forall \mu \in H^{-1 / 2}(\Gamma), \\
\mathcal{W} \psi(\mathbf{x}):=-\partial_{\nu(\mathbf{x})} \int_{\Gamma} \partial_{\nu(\mathbf{y})} E(\mathbf{x}, \mathbf{y}) \psi(\mathbf{y}) \mathrm{d}_{s} \mathbf{y} \quad \forall(\text { a.e. }) \mathbf{x} \in \Gamma, \forall \psi \in H^{1 / 2}(\Gamma),
\end{gathered}
$$

where $\partial_{\nu(\mathbf{x})}$ stands for the normal derivative operator at $\mathbf{x} \in \Gamma$.

Next, we observe from the definition of $\varphi$ and $\kappa$ that the unknown $\varphi$ belongs to the space $H_{0}^{1 / 2}(\Gamma):=\left\{\psi \in H^{1 / 2}(\Gamma): \quad\langle 1, \psi\rangle=0\right\}$, and, according to the behaviour of $u$ at infinity, there holds $\gamma \in H_{0}^{-1 / 2}(\Gamma):=\left\{\mu \in H^{-1 / 2}(\Gamma):\langle\mu, 1\rangle=0\right\}$. Hence, incorporating the transmission condition given by $\gamma=\lambda$ on $\Gamma$ (continuity of the normal derivative of $u$ ), the boundary integral equations (2.4) can be reformulated as the system: find $(\varphi, \gamma) \in H_{0}^{1 / 2}(\Gamma) \times H_{0}^{-1 / 2}(\Gamma)$ such that

$$
\begin{array}{ccccc}
\langle\mathcal{W} \varphi, \psi\rangle-\left\langle\left(\frac{1}{2} \mathcal{I}-\mathcal{K}^{\prime}\right) \gamma, \psi\right\rangle & =-\langle\lambda, \psi\rangle & & \forall \psi \in H_{0}^{1 / 2}(\Gamma), \\
\left\langle\mu,\left(\frac{1}{2} \mathcal{I}-\mathcal{K}\right) \varphi\right\rangle+\quad\langle\mu, \mathcal{V} \gamma\rangle & =0 & & \forall \mu \in H_{0}^{-1 / 2}(\Gamma),
\end{array}
$$

together with the a posteriori computation of the constant $\kappa$ :

$$
\kappa=-\frac{1}{|\Gamma|}\left\{\left\langle 1,\left(\frac{1}{2} \mathcal{I}-\mathcal{K}\right) \varphi\right\rangle+\langle 1, \mathcal{V} \gamma\rangle\right\} .
$$

The analysis of (2.5) and its discrete counterpart below will strongly depend on the symmetry and ellipticity properties of $\mathcal{V}$ and $\mathcal{W}$, which are given by

$$
\begin{aligned}
\langle\mu, \mathcal{V} \gamma\rangle & =\langle\gamma, \mathcal{V} \mu\rangle & & \forall \mu, \gamma \in H^{-1 / 2}(\Gamma), \\
\langle\mathcal{W} \varphi, \psi\rangle & =\langle\mathcal{W} \psi, \varphi\rangle & & \forall \varphi, \psi \in H^{1 / 2}(\Gamma), \\
\langle\mu, \mathcal{V} \mu\rangle & \geq C\|\mu\|_{-1 / 2, \Gamma}^{2} & & \forall \mu \in H_{0}^{-1 / 2}(\Gamma), \\
\langle\mathcal{W} \psi, \psi\rangle & \geq C\|\psi\|_{1 / 2, \Gamma}^{2} & & \forall \psi H_{0}^{1 / 2}(\Gamma) .
\end{aligned}
$$


2.2. The LDG formulation in the interior domain. We now let $\mathcal{T}_{h}$ be a shaperegular triangulation of $\bar{\Omega}$ (with possible hanging nodes) made up of straight triangles $K$ with diameter $h_{K}$ and unit outward normal to $\partial K$ given by $\nu_{K}$. As usual, the index $h$ also denotes $h:=\max _{K \in \mathcal{T}_{h}} h_{K}$. Then, the edges of $\mathcal{T}_{h}$ are defined as follows. An interior edge of $\mathcal{T}_{h}$ is the (nonempty) interior of $\partial K \cap \partial K^{\prime}$, where $K$ and $K^{\prime}$ are two adjacent elements of $\mathcal{T}_{h}$. Similarly, a boundary edge of $\mathcal{T}_{h}$ is the (nonempty) interior of $\partial K \cap \Gamma_{0}$ or $\partial K \cap \Gamma$, where $K$ is a boundary element of $\mathcal{T}_{h}$. For each edge $e, h_{e}$ represents its length. In addition, we define $\mathcal{E}(K):=$ edges of $K, \mathcal{E}_{h}^{\text {int }}$ : list of interior edges (counted only once), $\mathcal{E}_{h}^{\Gamma}$ : list of edges on $\Gamma$, $\mathcal{E}_{h}^{\Gamma_{0}}$ : list of edges on $\Gamma_{0}$, and $I_{h}$ : interior grid generated by the triangulation, that is, $I_{h}:=\bigcup\left\{e: e \in \mathcal{E}_{h}^{\text {int }}\right\}$. Also, we let $\Gamma_{h}^{0}$ and $\Gamma_{h}$ be the boundaries $\Gamma_{0}$ and $\Gamma$, respectively, divided into edges.

In what follows we assume that $\mathcal{T}_{h}$ is of bounded variation, which means that there exists $l>1$, independent of the meshsize $h$, such that $l^{-1} \leq \frac{h_{K}}{h_{K^{\prime}}} \leq l$ for each pair $K, K^{\prime} \in \mathcal{T}_{h}$ sharing an interior edge. We note that the hypotheses on the triangulation imply that the cardinality of $\mathcal{E}(K)$ is uniformly bounded, and that for each $e \in \mathcal{E}(K)$ there holds $h_{K} \leq C l h_{e}$.

In order to introduce the LDG approach, we first multiply the partial differential equations in (2.1) by smooth test functions $\tau$ and $v$, respectively, and integrate by parts over each $K \in \mathcal{T}_{h}$. Then, the basic idea is to consider a discrete setting of the resulting local conservation laws, but with the traces of $\sigma$ and $u$ on the boundary $\partial K$ of each $K \in \mathcal{T}_{h}$ being replaced by suitable numerical approximations $\bar{\sigma}$ and $\bar{u}$, respectively, which are named numerical fluxes.

To this end, given $m \in \mathbb{N}$, we define the finite element spaces

$$
V_{h}:=\prod_{K \in \mathcal{T}_{h}} P(K) \quad \text { and } \quad \boldsymbol{\Sigma}_{h}:=\prod_{K \in \mathcal{T}_{h}} \mathbf{P}(K)
$$

where $P(K):=P_{m}(K)$ and $\mathbf{P}(K):=\mathbf{P}_{r}(K)$, with $r=m$ or $r=m-1$. Hereafter, given an integer $k \geq 0$ and a domain $S \subseteq \mathbb{R}^{2}, P_{k}(S)$ denotes the space of polynomials of degree at most $k$ on $S$. Also, given $v:=\left\{v_{K}\right\}_{K \in \mathcal{T}_{h}} \in V_{h}$ and $\tau:=\left\{\tau_{K}\right\}_{K \in \mathcal{T}_{h}} \in \boldsymbol{\Sigma}_{h}$, the components $v_{K}$ and $\tau_{K}$ coincide with the restrictions $\left.v\right|_{K}$ and $\left.\tau\right|_{K}$, when $v$ and $\tau$ are identified as elements in $L^{2}(\Omega)$ and $\mathbf{L}^{2}(\Omega)$, respectively. Further, when no confusion arises, we omit the subscript $K$ and just write $v$ and $\tau$.

Hence, we consider the following formulation: find $\left(\sigma_{h}, u_{h}\right) \in \boldsymbol{\Sigma}_{h} \times V_{h}$ such that for each $K \in \mathcal{T}_{h}$ there holds

$$
\begin{array}{lll}
\int_{K} \sigma_{h} \cdot \tau+\int_{K} u_{h}(\operatorname{div} \tau)-\int_{\partial K} \bar{u}\left(\tau \cdot \nu_{K}\right) & =0 & \forall \tau \in \boldsymbol{\Sigma}_{h}, \\
\int_{K} \sigma_{h} \cdot \nabla v-\int_{\partial K}\left(\bar{\sigma} \cdot \nu_{K}\right) v & =\int_{K} f v & \forall v \in V_{h},
\end{array}
$$

where the numerical fluxes $\bar{u}$ and $\bar{\sigma}$, usually depending on $u_{h}, \sigma_{h}$, and the boundary conditions are chosen so that some compatibility conditions are satisfied. 
The explicit definitions of these fluxes require the introduction of further notation. Given $s>1 / 2$, we now let

$$
\begin{aligned}
H^{s}\left(\mathcal{T}_{h}\right) & :=\prod_{K \in \mathcal{T}_{h}} H^{s}(K), \quad L^{2}\left(I_{h}\right):=\prod_{e \in \mathcal{E}_{h}^{\text {int }}} L^{2}(e), \\
L^{2}\left(I_{h} \cup \Gamma_{h}^{0} \cup \Gamma_{h}\right) & =\prod_{e \in \mathcal{E}_{h}^{\text {int }} \cup \mathcal{E}_{h}^{\Gamma_{0}} \cup \mathcal{E}_{h}^{\Gamma}} L^{2}(e), \quad P_{0}\left(I_{h}\right):=\prod_{e \in \mathcal{E}_{h}^{\text {int }}} P_{0}(e), \\
P_{0}\left(I_{h} \cup \Gamma_{h}^{0}\right) & :=\prod_{e \in \mathcal{E}_{h}^{\text {int }} \cup \mathcal{E}_{h}^{\Gamma_{0}^{0}}} P_{0}(e) \quad \text { and } \quad P_{0}\left(\Gamma_{h}\right):=\prod_{e \in \mathcal{E}_{h}^{\Gamma}} P_{0}(e) .
\end{aligned}
$$

An analogue remark to the one given before, concerning components and restrictions of the elements in $V_{h}$ and $\boldsymbol{\Sigma}_{h}$, is valid here for each one of the above product spaces. Also, we will not use any symbol for the trace on edges, provided it is clear from which side of an interior edge we are taking the trace. Hence, given $v \in H^{1}\left(\mathcal{T}_{h}\right)$, we define the averages $\{v\} \in L^{2}\left(I_{h}\right)$ and jumps $\llbracket v \rrbracket \in \mathbf{L}^{2}\left(I_{h}\right)$ on the interior grid $I_{h}$ by

$$
\{v\}_{e}:=\frac{1}{2}\left(v_{K}+v_{K^{\prime}}\right) \quad \text { and } \quad \llbracket v \rrbracket_{e}:=v_{K} \nu_{K}+v_{K^{\prime}} \nu_{K^{\prime}} \quad \forall e \in \mathcal{E}(K) \cap \mathcal{E}\left(K^{\prime}\right) .
$$

Similarly, for vector valued functions $\tau \in \mathbf{H}^{1}\left(\mathcal{T}_{h}\right)$, we define $\{\tau\} \in \mathbf{L}^{2}\left(I_{h}\right)$ and $\llbracket \tau \rrbracket \in L^{2}\left(I_{h}\right)$ by

$$
\{\tau\}_{e}:=\frac{1}{2}\left(\tau_{K}+\tau_{K^{\prime}}\right) \quad \text { and } \quad \llbracket \tau \rrbracket_{e}:=\tau_{K} \cdot \nu_{K}+\tau_{K^{\prime}} \cdot \nu_{K^{\prime}} \quad \forall e \in \mathcal{E}(K) \cap \mathcal{E}\left(K^{\prime}\right) .
$$

In addition, we let $\alpha \in P_{0}\left(I_{h} \cup \Gamma_{h}^{0}\right)$ and $\beta \in \mathbf{P}_{0}\left(I_{h}\right)$ be given functions and assume that there exist $C, c_{0}, c_{1}>0$, independent of the grid, such that

$$
\max _{e \in \mathcal{E}_{h}^{\text {int }}}\left|\beta_{e}\right| \leq C \quad \text { and } \quad 0<c_{0} \leq h_{\mathcal{E}} \alpha \leq c_{1}
$$

where $h_{\mathcal{E}} \in P_{0}\left(I_{h} \cup \Gamma_{h}^{0}\right)$ is defined by $\left.h_{\mathcal{E}}\right|_{e}:=h_{e} \quad \forall e \in \mathcal{E}_{h}^{\text {int }} \cup \mathcal{E}_{h}^{\Gamma_{0}}$.

Then, using the approach in [8] and [28, the flux operators $\widehat{u}: H^{1}\left(\mathcal{T}_{h}\right) \rightarrow$ $L^{2}\left(I_{h} \cup \Gamma_{h}^{0} \cup \Gamma_{h}\right)$ and $\widehat{\sigma}: H^{1}\left(\mathcal{T}_{h}\right) \times \mathbf{H}^{1}\left(\mathcal{T}_{h}\right) \times L^{2}(\Gamma) \rightarrow \mathbf{L}^{2}\left(I_{h} \cup \Gamma_{h}^{0} \cup \Gamma_{h}\right)$ are defined componentwise as follows:

$$
\widehat{u}(v)_{e}:= \begin{cases}\{v\}_{e}+\beta_{e} \cdot \llbracket v \rrbracket_{e} & \text { if } \quad e \in \mathcal{E}_{h}^{\text {int }}, \\ 0 & \text { if } \quad e \in \mathcal{E}_{h}^{\Gamma_{0}}, \\ v_{e} & \text { if } \quad e \in \mathcal{E}_{h}^{\Gamma},\end{cases}
$$

for all $v \in H^{1}\left(\mathcal{T}_{h}\right)$, and

$$
\widehat{\sigma}(v, \tau, \xi)_{e}:= \begin{cases}\{\tau\}_{e}-\llbracket \tau \rrbracket_{e} \beta_{e}-\alpha_{e} \llbracket v \rrbracket & \text { if } e \in \mathcal{E}_{h}^{\text {int }}, \\ \tau_{e}-\alpha_{e} v_{e} \nu_{e} & \text { if } e \in \mathcal{E}_{h}^{\Gamma_{0}}, \\ \xi \nu_{e} & \text { if } e \in \mathcal{E}_{h}^{\Gamma},\end{cases}
$$

for all $(v, \tau, \xi) \in H^{1}\left(\mathcal{T}_{h}\right) \times \mathbf{H}^{1}\left(\mathcal{T}_{h}\right) \times L^{2}(\Gamma)$.

We now introduce a discrete approximation of $\lambda$. To this end, we let $\Gamma_{\widetilde{h}}$ be a second partition of $\Gamma$, independent of the partition $\Gamma_{h}$ inherited from $\mathcal{T}_{h}$, and denote by $\mathcal{E}_{\widetilde{h}}^{\Gamma}$ the corresponding list of edges $\widetilde{e}$. Then, given $\widetilde{k} \in \mathbb{N}$, we define the 
subspaces:

$$
\begin{gathered}
X_{\widetilde{h}}:=\left\{\xi_{\widetilde{h}} \in L^{2}(\Gamma):\left.\quad \xi_{\widetilde{h}}\right|_{\tilde{e}} \in P_{\widetilde{k}}(\widetilde{e}) \forall \widetilde{e} \in \mathcal{E}_{\widetilde{h}}^{\Gamma}\right\}, \\
X_{\widetilde{h}}^{0}=\left\{\xi_{\widetilde{h}} \in X_{\widetilde{h}}: \quad \int_{\Gamma} \xi_{\widetilde{h}}=0\right\},
\end{gathered}
$$

and let $\lambda_{\tilde{h}} \in X_{\widetilde{h}}^{0}$ be an unknown approximating $\lambda$. Hence, we consider (2.8) with numerical fluxes $\bar{u}:=\widehat{u}\left(u_{h}\right)$ and $\bar{\sigma}:=\widehat{\sigma}\left(u_{h}, \sigma_{h}, \lambda_{\tilde{h}}\right)$, integrate by parts the second term of its first equation, and obtain the formulation: find $\left(\sigma_{h}, u_{h}, \lambda_{\tilde{h}}\right) \in$ $\Sigma_{h} \times V_{h} \times X_{\widetilde{h}}^{0}$ such that for each $K \in \mathcal{T}_{h}$ there holds

$$
\begin{aligned}
& \int_{K} \sigma_{h} \cdot \tau-\int_{K} \nabla u_{h} \cdot \tau+\int_{\partial K}\left(u_{h}-\bar{u}\right) \tau \cdot \nu_{K} \quad=0 \quad \forall \tau \in \boldsymbol{\Sigma}_{h}, \\
& \int_{K} \sigma_{h} \cdot \nabla v-\int_{\partial K \cap\left(\Omega \cup \Gamma_{0}\right)}\left(\bar{\sigma} \cdot \nu_{K}\right) v-\int_{\partial K \cap \Gamma} \lambda_{\widetilde{h}} v=\int_{K} f v \quad \forall v \in V_{h} .
\end{aligned}
$$

Next, we sum up (2.11) over all $K \in \mathcal{T}_{h}$, apply well-known algebraic identities, and arrive at the global LDG formulation: find $\left(\sigma_{h}, u_{h}, \lambda_{\widetilde{h}}\right) \in \boldsymbol{\Sigma}_{h} \times V_{h} \times X_{\widetilde{h}}^{0}$ such that

$$
\begin{array}{lll}
\int_{\Omega} \sigma_{h} \cdot \tau-\left\{\int_{\Omega} \nabla_{h} u_{h} \cdot \tau-S\left(u_{h}, \tau\right)\right\} & =0 & \forall \tau \in \boldsymbol{\Sigma}_{h}, \\
\left\{\int_{\Omega} \nabla_{h} v \cdot \sigma_{h}-S\left(v, \sigma_{h}\right)\right\}+\alpha\left(u_{h}, v\right)-\int_{\Gamma} \lambda_{\widetilde{h}} v & =\int_{\Omega} f v & \forall v \in V_{h},
\end{array}
$$

where $\nabla_{h}$ stands for the piecewise defined gradient, and $S: H^{1}\left(\mathcal{T}_{h}\right) \times \mathbf{H}^{1}\left(\mathcal{T}_{h}\right) \rightarrow \mathbb{R}$ and $\alpha: H^{1}\left(\mathcal{T}_{h}\right) \times H^{1}\left(\mathcal{T}_{h}\right) \rightarrow \mathbb{R}$ are the bilinear forms defined by

$S(w, \tau):=\int_{I_{h}} \llbracket w \rrbracket \cdot(\{\tau\}-\llbracket \tau \rrbracket \beta)+\int_{\Gamma_{0}} w(\tau \cdot \nu) \quad \forall(w, \tau) \in H^{1}\left(\mathcal{T}_{h}\right) \times \mathbf{H}^{1}\left(\mathcal{T}_{h}\right)$,

and

$$
\alpha(w, v):=\int_{I_{h}} \alpha \llbracket w \rrbracket \cdot \llbracket v \rrbracket+\int_{\Gamma_{0}} \alpha w v \quad \forall(w, v) \in H^{1}\left(\mathcal{T}_{h}\right) \times H^{1}\left(\mathcal{T}_{h}\right),
$$

with the traces of $w, v$, and $\tau$ on $\Gamma_{0}$ being certainly defined elementwise, not globally.

We remark that if $w \in H^{t}(\Omega)$, with $t>1$, then $\llbracket w \rrbracket=0$ on $I_{h}$. Additionally, if $w=0$ on $\Gamma_{0}$, then $S(w, \tau)=0$ for all $\tau \in \mathbf{H}^{1}\left(\mathcal{T}_{h}\right)$. Furthermore, if $-\Delta w=f$ in $\Omega$ and $t>3 / 2$, then simple computations show, with $\sigma:=\nabla w$ and $\lambda:=\partial_{\nu} w$, that there holds

$$
\begin{array}{llll}
\int_{\Omega} \sigma \cdot \tau-\left\{\int_{\Omega} \nabla_{h} w \cdot \tau-S(w, \tau)\right\} & =0 & \forall \tau \in \mathbf{H}^{1}\left(\mathcal{T}_{h}\right), \\
\left\{\int_{\Omega} \nabla_{h} v \cdot \sigma-S(v, \sigma)\right\}+\alpha(w, v)-\int_{\Gamma} \lambda v & =\int_{\Omega} f v & \forall v \in H^{1}\left(\mathcal{T}_{h}\right) .
\end{array}
$$

We end this section by observing that the definitions of averages, jumps, and fluxes, as well as the bilinear forms $S$ and $\alpha$, can be extended to $(v, w, \tau) \in$ $H^{1 / 2+\epsilon}\left(\mathcal{T}_{h}\right) \times H^{1 / 2+\epsilon}\left(\mathcal{T}_{h}\right) \times \mathbf{H}^{1 / 2+\epsilon}\left(\mathcal{T}_{h}\right)$ for any $\epsilon>0$. 
2.3. The coupled LDG-BEM scheme. We now let $\Gamma_{\widehat{h}}$ be a third partition of $\Gamma$, independent of the partition $\Gamma_{h}$ inherited from $\mathcal{T}_{h}$, and denote by $\mathcal{E}_{\widehat{h}}^{\Gamma}$ the corresponding list of edges $\widehat{e}$. In addition, given $\widehat{k} \in \mathbb{N}$, we introduce the boundary element subspaces:

$$
\begin{gathered}
Y_{\widehat{h}}:=\left\{\psi_{\widehat{h}} \in \mathcal{C}(\Gamma):\left.\quad \psi_{\widehat{h}}\right|_{\widehat{e}} \in P_{\widehat{k}}(\widehat{e}) \forall \widehat{e} \in \mathcal{E}_{\widehat{h}}^{\Gamma}\right\}, \\
Y_{\widehat{h}}^{0}=\left\{\psi_{\widehat{h}} \in Y_{\widehat{h}}: \quad \int_{\Gamma} \psi_{\widehat{h}}=0\right\} \\
Z_{\widehat{h}}:=\left\{\mu_{\widehat{h}} \in L^{2}(\Gamma):\left.\quad \mu_{\widehat{h}}\right|_{\widehat{e}} \in P_{\widehat{k}-1}(\widehat{e}) \forall \widehat{e} \in \mathcal{E}_{\widehat{h}}^{\Gamma}\right\}, \\
Z_{\widehat{h}}^{0}=\left\{\mu_{\widehat{h}} \in Z_{\widehat{h}}: \quad \int_{\Gamma} \mu_{\widehat{h}}=0\right\} .
\end{gathered}
$$

Then, we substitute $\lambda$ by $\lambda_{\widetilde{h}}$ in the right-hand side of the boundary integral equations (2.5) and define its discrete version as follows: find $\left(\varphi_{\widehat{h}}, \gamma_{\widehat{h}}\right) \in Y_{\widehat{h}}^{0} \times Z_{\widehat{h}}^{0}$ such that

$$
\begin{array}{ccccc}
\left\langle\mathcal{W} \varphi_{\widehat{h}}, \psi\right\rangle-\left\langle\left(\frac{1}{2} \mathcal{I}-\mathcal{K}^{\prime}\right) \gamma_{\widehat{h}}, \psi\right\rangle & =-\left\langle\lambda_{\widehat{h}}, \psi\right\rangle & & \forall \psi \in Y_{\widehat{h}}^{0}, \\
\left\langle\mu,\left(\frac{1}{2} \mathcal{I}-\mathcal{K}\right) \varphi_{\widehat{h}}\right\rangle+\quad\left\langle\mu, \mathcal{V} \gamma_{\widehat{h}}\right\rangle & =0 & & \forall \mu \in Z_{\widehat{h}}^{0} .
\end{array}
$$

In addition, the transmission condition given by the first equation in (2.2) is imposed weakly, at the discrete level, as follows:

$$
\left\langle\xi, u_{h}\right\rangle-\left\langle\xi, \varphi_{\widehat{h}}\right\rangle=0 \quad \forall \xi \in X_{\widetilde{h}}^{0} .
$$

Therefore, (2.12), (2.17), and (2.18) lead to our coupled LDG-BEM formulation: find $\left(\sigma_{h}, u_{h}, \lambda_{\widetilde{h}}, \varphi_{\widehat{h}}, \gamma_{\widehat{h}}\right) \in \Sigma_{h} \times V_{h} \times X_{\widetilde{h}}^{0} \times Y_{\widehat{h}}^{0} \times Z_{\widehat{h}}^{0}$ such that

$$
\begin{aligned}
\int_{\Omega} \sigma_{h} \cdot \tau-\rho\left(u_{h}, \tau\right) & =0, \\
\rho\left(v, \sigma_{h}\right)+\alpha\left(u_{h}, v\right)-\left\langle\lambda_{\tilde{h}}, v\right\rangle & =\int_{\Omega} f v, \\
\left\langle\xi, u_{h}\right\rangle & =0, \\
\left\langle\lambda_{\widetilde{h}}, \psi\right\rangle+\left\langle\mathcal{W} \varphi_{\widehat{h}}, \psi\right\rangle-\left\langle\left(\frac{1}{2} \mathcal{I}-\mathcal{K}^{\prime}\right) \gamma_{\widehat{h}}, \psi\right\rangle & =0, \\
\left\langle\mu,\left(\frac{1}{2} \mathcal{I}-\mathcal{K}\right) \varphi_{\widehat{h}}\right\rangle+\left\langle\mu, \mathcal{V} \gamma_{\widehat{h}}\right\rangle & =0,
\end{aligned}
$$

for all $(\tau, v, \xi, \psi, \mu) \in \boldsymbol{\Sigma}_{h} \times V_{h} \times X_{\widetilde{h}}^{0} \times Y_{\widehat{h}}^{0} \times Z_{\widehat{h}}^{0}$, where $\rho: H^{1}\left(\mathcal{T}_{h}\right) \times \mathbf{H}^{1}\left(\mathcal{T}_{h}\right) \rightarrow \mathbb{R}$ is the bilinear form defined by

$$
\rho(v, \tau):=\int_{\Omega} \nabla_{h} v \cdot \tau-S(v, \tau) \quad \forall(v, \tau) \in H^{1}\left(\mathcal{T}_{h}\right) \times \mathbf{H}^{1}\left(\mathcal{T}_{h}\right) .
$$

It is important to remark here that $\lambda_{\tilde{h}}$ plays the role of a mortar-type auxiliary unknown gluing the LDG and BEM formulations in a suitable way, as we will see below in Section 3, where the unique solvability and stability of (2.19) is proved. On the contrary, the eventual replacement of $\lambda_{\widetilde{h}}$ in (2.19) by the exterior approximation $\gamma_{\widehat{h}}$ of the normal derivative of $u$ would yield an overdetermined coupled formulation with 5 equations and 4 unknowns. This would certainly add further difficulties for computing the solution (whenever it exists) of the resulting linear system. In particular, determining the linearly independent equations could be expensive and numerically unstable. 
On the other hand, we also note that an element-by-element formulation for the LDG part, suitable for computational implementation, is straightforward from (2.19). In addition, the constant $\kappa$ needed to compute the behaviour at infinity of the solution $u$ can be approximated a posteriori either by

$$
\kappa_{\widehat{h}}=-\frac{1}{|\Gamma|} \int_{\Gamma}\left\{\left(\frac{1}{2} \mathcal{I}-\mathcal{K}\right) \varphi_{\widehat{h}}+\mathcal{V}_{\widehat{h}}\right\} \quad \text { or } \quad \kappa_{h}:=\frac{1}{|\Gamma|} \int_{\Gamma} u_{h}
$$

2.4. The reduced coupled LDG-BEM scheme. In order to study the solvability and stability of (2.19), and following the usual analysis for LDG methods, we derive in this section an equivalent reduced formulation. For this purpose, we now let $\mathbf{S}_{h}: H^{1}\left(\mathcal{T}_{h}\right) \rightarrow \boldsymbol{\Sigma}_{h}$ be the linear operator associated to the bilinear form $S$ when restricted to $H^{1}\left(\mathcal{T}_{h}\right) \times \boldsymbol{\Sigma}_{h}$. In other words, given $w \in H^{1}\left(\mathcal{T}_{h}\right), \mathbf{S}_{h}(w)$ is the unique element in $\boldsymbol{\Sigma}_{h}$ satisfying

$$
\int_{\Omega} \mathbf{S}_{h}(w) \cdot \tau=S(w, \tau) \quad \forall \tau \in \mathbf{\Sigma}_{h}
$$

According to a remark given in the previous subsection, we observe that if $w$ is such that $w \in H^{t}(\Omega)$, with $t>1$, and $w=0$ on $\Gamma_{0}$, then $\mathbf{S}_{h}(w)=0$.

Next, we let $B_{h}: H^{1}\left(\mathcal{T}_{h}\right) \times H^{1}\left(\mathcal{T}_{h}\right) \rightarrow \mathbb{R}$ be the bilinear form defined by

$$
B_{h}(w, v):=\alpha(w, v)+\int_{\Omega}\left(\nabla_{h} w-\mathbf{S}_{h}(w)\right) \cdot\left(\nabla_{h} v-\mathbf{S}_{h}(v)\right) \quad \forall w, v \in H^{1}\left(\mathcal{T}_{h}\right),
$$

and denote by $\Pi_{\boldsymbol{\Sigma}_{h}}$ the $\mathbf{L}^{2}(\Omega)$-projection onto $\boldsymbol{\Sigma}_{h}$. We have the following technical result.

Lemma 2.1. Let $w \in H^{t}(\Omega)$, with $t>3 / 2$, such that $w=0$ on $\Gamma_{0}$ and $\Delta w \in$ $L^{2}(\Omega)$. Then

$B_{h}(w, v)=\int_{\Omega}(-\Delta w) v+S\left(v, \nabla w-\Pi_{\boldsymbol{\Sigma}_{h}}(\nabla w)\right)+\int_{\Gamma} v(\nabla w \cdot \nu) \quad \forall v \in H^{1}\left(\mathcal{T}_{h}\right)$.

Proof. Because of the hypotheses on $w$ we easily find that $\llbracket w \rrbracket=0$ on $I_{h}, \mathbf{S}_{h}(w)=0$, and $\alpha(w, v)=0$ for all $v \in H^{1}\left(\mathcal{T}_{h}\right)$. It follows that

$$
\begin{aligned}
B_{h}(w, v) & =\int_{\Omega} \nabla w \cdot \nabla_{h} v-\int_{\Omega} \nabla w \cdot \mathbf{S}_{h}(v) \\
& =\sum_{K \in \mathcal{T}_{h}} \int_{K} \nabla w \cdot \nabla v-\int_{\Omega} \Pi_{\mathbf{\Sigma}_{h}}(\nabla w) \cdot \mathbf{S}_{h}(v) \\
& =\sum_{K \in \mathcal{T}_{h}}\left\{\int_{K}(-\Delta w) v+\int_{\partial K}\left(\nabla w \cdot \nu_{K}\right) v\right\}-S\left(v, \Pi_{\mathbf{\Sigma}_{h}}(\nabla w)\right) .
\end{aligned}
$$

In addition, since $\Delta w \in L^{2}(\Omega)$, we have $\nabla w \in H($ div $; \Omega)$ and hence $\llbracket \nabla w \rrbracket=0$. Therefore, we deduce that

$$
\begin{aligned}
& \sum_{K \in \mathcal{T}_{h}} \int_{\partial K}\left(\nabla w \cdot \nu_{K}\right) v=\int_{I_{h}}\{v\} \llbracket \nabla w \rrbracket+\int_{I_{h}} \llbracket v \rrbracket \cdot\{\nabla w\}+\int_{\partial \Omega} v(\nabla w \cdot \nu) \\
= & \int_{I_{h}} \llbracket v \rrbracket \cdot\{\nabla w\}+\int_{\Gamma_{0}} v(\nabla w \cdot \nu)+\int_{\Gamma} v(\nabla w \cdot \nu)=S(v, \nabla w)+\int_{\Gamma} v(\nabla w \cdot \nu),
\end{aligned}
$$

which, together with the previous expression for $B_{h}(w, v)$, completes the proof.

The equivalence between (2.12) and a reduced problem involving $B_{h}$ is now established. 
Lemma 2.2. Let $\left(\sigma_{h}, u_{h}, \lambda_{\widetilde{h}}\right) \in \boldsymbol{\Sigma}_{h} \times V_{h} \times X_{\widetilde{h}}^{0}$ be a solution of (2.12). Then there holds

$$
B_{h}\left(u_{h}, v\right)=\int_{\Omega} f v+\int_{\Gamma} \lambda_{\tilde{h}} v \quad \forall v \in V_{h} .
$$

Conversely, if $\left(u_{h}, \lambda_{\tilde{h}}\right) \in V_{h} \times X_{\widetilde{h}}^{0}$ satisfies (2.22) and $\sigma_{h}:=\nabla_{h} u_{h}-\mathbf{S}_{h}\left(u_{h}\right)$, then $\left(\sigma_{h}, u_{h}, \lambda_{\widetilde{h}}\right)$ is a solution of (2.12).

Proof. Let $\left(\sigma_{h}, u_{h}, \lambda_{\tilde{h}}\right) \in \Sigma_{h} \times V_{h} \times X_{\widetilde{h}}^{0}$ be a solution of (2.12). According to the first equation of (2.12) and the definition of $\mathbf{S}_{h}$, we can write

$$
\int_{\Omega} \sigma_{h} \cdot \tau-\int_{\Omega}\left(\nabla_{h} u_{h}-\mathbf{S}_{h}\left(u_{h}\right)\right) \cdot \tau=0 \quad \forall \tau \in \mathbf{\Sigma}_{h},
$$

which, noting that $\nabla_{h} u_{h} \in \mathbf{\Sigma}_{h}$, yields $\sigma_{h}=\nabla_{h} u_{h}-\mathbf{S}_{h}\left(u_{h}\right)$. Then, replacing this expression in the second equation of (2.12) we obtain (2.22). The converse result follows readily. We omit details.

At this point we observe that for all $w, v \in V_{h}$ we can write

$$
\begin{aligned}
B_{h}(w, v)= & \alpha(w, v)+\int_{\Omega} \nabla_{h} w \cdot \nabla_{h} v+\int_{\Omega} \mathbf{S}_{h}(w) \cdot \mathbf{S}_{h}(v) \\
& -S\left(w, \nabla_{h} v\right)-S\left(v, \nabla_{h} w\right)=: B_{h}^{0}(w, v),
\end{aligned}
$$

where $B_{h}^{0}$ is a bilinear form defined in $H^{2}\left(\mathcal{T}_{h}\right) \times H^{2}\left(\mathcal{T}_{h}\right)$. Because of the coincidence in the discrete spaces, $B_{h}^{0}$ also serves for a primal formulation of the LDG (see [2]). Furthermore, we note that if $w \in H^{t}(\Omega)$, with $t>3 / 2$, is such that $w=0$ on $\Gamma_{0}$ and $\Delta w \in L^{2}(\Omega)$, then

$$
B_{h}^{0}(w, v)=\int_{\Omega}(-\Delta w) v+\int_{\Gamma} v(\nabla w \cdot \nu) \quad \forall v \in H^{2}\left(\mathcal{T}_{h}\right) .
$$

In the forthcoming analysis we utilize the bilinear form $B_{h}$, which adds an inconsistency term depending on $S$ (see Lemma 2.1) but is defined in the larger space $H^{1}\left(\mathcal{T}_{h}\right) \times H^{1}\left(\mathcal{T}_{h}\right)$.

We now let $\mathbf{D}$ and $\overline{\mathbf{D}}:\left(H^{1 / 2}(\Gamma) \times H^{-1 / 2}(\Gamma)\right) \times\left(H^{1 / 2}(\Gamma) \times H^{-1 / 2}(\Gamma)\right) \rightarrow \mathbb{R}$ be the bilinear forms obtained after adding and subtracting the equations in (2.5), that is,

$$
\mathbf{D}((\varphi, \gamma),(\psi, \mu)):=\langle\mathcal{W} \varphi, \psi\rangle-\left\langle\left(\frac{1}{2} \mathcal{I}-\mathcal{K}^{\prime}\right) \gamma, \psi\right\rangle+\left\langle\mu,\left(\frac{1}{2} \mathcal{I}-\mathcal{K}\right) \varphi\right\rangle+\langle\mu, \mathcal{V} \gamma\rangle
$$

and

$$
\overline{\mathbf{D}}((\varphi, \gamma),(\psi, \mu)):=\langle\mathcal{W} \varphi, \psi\rangle-\left\langle\left(\frac{1}{2} \mathcal{I}-\mathcal{K}^{\prime}\right) \gamma, \psi\right\rangle-\left\langle\mu,\left(\frac{1}{2} \mathcal{I}-\mathcal{K}\right) \varphi\right\rangle-\langle\mu, \mathcal{V} \gamma\rangle
$$

for all $(\varphi, \gamma),(\psi, \mu) \in H^{1 / 2}(\Gamma) \times H^{-1 / 2}(\Gamma)$.

According to the mapping properties of the boundary integral operators we observe that $\mathbf{D}$ and $\overline{\mathbf{D}}$ are bounded. In addition, as a consequence of (2.6), we deduce that $\overline{\mathbf{D}}$ is symmetric and that $\mathbf{D}$ is strongly elliptic on $H_{0}^{1 / 2}(\Gamma) \times H_{0}^{-1 / 2}(\Gamma)$. In particular, the ellipticity of $\mathbf{D}$ allows us to define the linear operator $\mathbf{g}_{\widehat{h}}: H^{-1 / 2}(\Gamma) \rightarrow$ $Y_{\widehat{h}}^{0} \times Z_{\widehat{h}}^{0}$, where, given $\xi \in H^{-1 / 2}(\Gamma), \mathbf{g}_{\widehat{h}}(\xi):=\left(\mathbf{g}_{\widehat{h}}^{1}(\xi), \mathbf{g}_{\widehat{h}}^{2}(\xi)\right)$ is the unique element in $Y_{\widehat{h}}^{0} \times Z_{\widehat{h}}^{0}$ such that

$$
\mathbf{D}\left(\mathbf{g}_{\widehat{h}}(\xi),(\psi, \mu)\right)=\langle\xi, \psi\rangle \quad \forall(\psi, \mu) \in Y_{\widehat{h}}^{0} \times Z_{\widehat{h}}^{0},
$$


or, equivalently, with $\overline{\mathbf{D}}$ instead of $\mathbf{D}$,

$$
\overline{\mathbf{D}}\left(\mathbf{g}_{\widehat{h}}(\xi),(\psi, \mu)\right)=\langle\xi, \psi\rangle \quad \forall(\psi, \mu) \in Y_{\widehat{h}}^{0} \times Z_{\widehat{h}}^{0} .
$$

Thanks to the lifting operator $\mathbf{g}_{\widehat{h}}$, we can now establish the following equivalence result.

Lemma 2.3. Let $\left(\sigma_{h}, u_{h}, \lambda_{\widetilde{h}}, \varphi_{\widehat{h}}, \gamma_{\widehat{h}}\right) \in \boldsymbol{\Sigma}_{h} \times V_{h} \times X_{\widetilde{h}}^{0} \times Y_{\widehat{h}}^{0} \times Z_{\widehat{h}}^{0}$ be a solution of (2.19). Then there holds

$$
\begin{array}{lll}
B_{h}\left(u_{h}, v\right)-\left\langle\lambda_{\widetilde{h}}, v\right\rangle & =\int_{\Omega} f v & \forall v \in V_{h}, \\
\left\langle\xi, u_{h}\right\rangle+\left\langle\xi, \mathbf{g}_{\widehat{h}}^{1}\left(\lambda_{\widetilde{h}}\right)\right\rangle=0 & \forall \xi \in X_{\widetilde{h}}^{0} .
\end{array}
$$

Conversely, if $\left(u_{h}, \lambda_{\widetilde{h}}\right) \in V_{h} \times X_{\widetilde{h}}^{0}$ satisfies (2.25) and $\sigma_{h}:=\nabla_{h} u_{h}-\mathbf{S}_{h}\left(u_{h}\right)$, $\left(\varphi_{\widehat{h}}, \gamma_{\widehat{h}}\right):=\mathbf{g}_{\widehat{h}}\left(-\lambda_{\tilde{h}}\right)$, then $\left(\sigma_{h}, u_{h}, \lambda_{\tilde{h}}, \varphi_{\widehat{h}}, \gamma_{\widehat{h}}\right)$ is a solution of (2.19).

Proof. Let $\left(\sigma_{h}, u_{h}, \lambda_{\widetilde{h}}, \varphi_{\widehat{h}}, \gamma_{\widehat{h}}\right) \in \Sigma_{h} \times V_{h} \times X_{\widetilde{h}}^{0} \times Y_{\widehat{h}}^{0} \times Z_{\widehat{h}}^{0}$ be a solution of (2.19). It is easy to realize that the last two equations in (2.19) imply that

$$
\left(\varphi_{\widehat{h}}, \gamma_{\widehat{h}}\right)=\mathbf{g}_{\widehat{h}}\left(-\lambda_{\widetilde{h}}\right):=\left(\mathbf{g}_{\widehat{h}}^{1}\left(-\lambda_{\widetilde{h}}\right), \mathbf{g}_{\widehat{h}}^{2}\left(-\lambda_{\widetilde{h}}\right)\right) .
$$

Therefore, replacing $\varphi_{\widehat{h}}$ by $\mathbf{g}_{\widehat{h}}^{1}\left(-\lambda_{\tilde{h}}\right)$ in the third equation of (2.19), and applying Lemma 2.2, we arrive at (2.25). The converse result follows from the definition of $\mathbf{g}_{\hat{h}}$ and Lemma 2.2. Further details are omitted.

\section{UNIQUE SOLVABILITY AND STABILITY}

In this section we prove the unique solvability and stability of (2.19) through the corresponding analysis of the equivalent reduced formulation (2.25). To this end, we follow $[8]$ and first introduce the seminorms

$$
|v|_{1, h}^{2}:=\left\|\nabla_{h} v\right\|_{0, \Omega}^{2}, \quad|v|_{*}^{2}:=\left\|h_{\mathcal{E}}^{-1 / 2} \llbracket v \rrbracket\right\|_{0, I_{h}}^{2}+\left\|h_{\mathcal{E}}^{-1 / 2} v\right\|_{0, \Gamma_{0}}^{2} \quad \forall v \in H^{1}\left(\mathcal{T}_{h}\right),
$$

and the norm

$$
\|v\|_{h}^{2}:=|v|_{1, h}^{2}+|v|_{*}^{2} \quad \forall v \in H^{1}\left(\mathcal{T}_{h}\right) .
$$

In addition, in order to deal with the mortar-type unknown $\lambda_{\widetilde{h}}$, we need to define the seminorm:

$$
|\xi|_{\widehat{h}}:=\sup _{0 \neq \psi_{\hat{h}} \in Y_{\widehat{h}}} \frac{\left\langle\xi, \psi_{\widehat{h}}\right\rangle}{\left\|\psi_{\widehat{h}}\right\|_{1 / 2, \Gamma}} \quad \forall \xi \in H^{-1 / 2}(\Gamma),
$$

which clearly satisfies $|\xi|_{\widehat{h}} \leq\|\xi\|_{-1 / 2, \Gamma}$ for all $\xi \in H^{-1 / 2}(\Gamma)$. Moreover, throughout the rest of the paper we assume that $|\cdot|_{\widehat{h}}$ is a norm in $X_{\widetilde{h}}$, which is equivalent to $\|\cdot\|_{-1 / 2, \Gamma}$ in $X_{\widetilde{h}}$.

In particular, we will show in Lemma 3.1 that the lower bound

$$
|\xi|_{\widehat{h}} \geq C\|\xi\|_{-1 / 2, \Gamma}
$$

holds for all $\xi \in X_{\widetilde{h}}$ if a technical condition on the mesh sizes is satisfied. Indeed, let us now assume that the partition $\Gamma_{\widetilde{h}}$ is uniformly regular, which means that there exists $c>0$, independent of $\widetilde{h}$, such that $|\widetilde{e}| \geq c \widetilde{h}$ for all $\widetilde{e} \in \mathcal{E}_{\widetilde{h}}^{\Gamma}$. This assumption yields the inverse inequality for $X_{\widetilde{h}}$, which says that for any real numbers $s$ and $t$ with $-1 / 2 \leq s \leq t \leq 0$, there exists $C>0$ such that

$$
\|\xi\|_{t, \Gamma} \leq C \widetilde{h}^{s-t}\|\xi\|_{s, \Gamma} \quad \forall \xi \in X_{\widetilde{h}}
$$


Also, we recall here the approximation property of the space $Y_{\widehat{h}}$, which establishes that for any $1 / 2<t \leq 3 / 2$ and for any $\psi \in H^{t}(\Gamma)$, there exists $\widehat{\psi}_{\widehat{h}} \in Y_{\widehat{h}}$ such that

$$
\left\|\psi-\widehat{\psi}_{\widehat{h}}\right\|_{1 / 2, \Gamma} \leq C \widehat{h}^{t-1 / 2}\|\psi\|_{t, \Gamma} .
$$

Then, we can prove the following result.

Lemma 3.1. There exist positive constants $C_{0}, \tilde{C}$, independent of the meshsizes, such that for each $\widehat{h} \leq C_{0} \widetilde{h}$ there holds

$$
|\xi|_{\widehat{h}} \geq \tilde{C}\|\xi\|_{-1 / 2, \Gamma} \quad \forall \xi \in X_{\widetilde{h}} .
$$

Proof. Given $\xi \in X_{\widetilde{h}}$, we let $z \in H^{1}(\Omega)$ be the unique weak solution of the boundary value problem

$$
-\Delta z+z=0 \quad \text { in } \Omega, \quad z=0 \quad \text { on } \Gamma_{0}, \partial_{\nu} z=\xi \text { on } \Gamma \text {. }
$$

Since $\xi \in H^{-1 / 2+\delta}(\Gamma)$ for some $\delta>1 / 2$, we have $z \in H^{1+\delta}(\Omega)$ and $\|z\|_{1+\delta, \Omega} \leq$ $c\|\xi\|_{-1 / 2+\delta, \Gamma}$. In addition, there also holds $\|z\|_{1, \Omega} \leq c\|\xi\|_{-1 / 2, \Gamma}$. Then, we let $\psi:=\left.z\right|_{\Gamma} \in H^{1 / 2+\delta}(\Gamma)$ and apply the approximation property (3.3) to deduce the existence of $\widehat{\psi}_{\widehat{h}} \in Y_{\widehat{h}}$ such that

$$
\left\|\psi-\widehat{\psi}_{\widehat{h}}\right\|_{1 / 2, \Gamma} \leq C \widehat{h}^{\delta}\|\psi\|_{1 / 2+\delta, \Gamma} .
$$

Next, using trace theorem and inverse inequality (3.2), we find that

$$
\begin{aligned}
\left\|\psi-\widehat{\psi}_{\widehat{h}}\right\|_{1 / 2, \Gamma} & \leq C \widehat{h}^{\delta}\|\psi\|_{1 / 2+\delta, \Gamma} \leq C \widehat{h}^{\delta}\|z\|_{1+\delta, \Omega} \\
& \leq C \widehat{h}^{\delta}\|\xi\|_{-1 / 2+\delta, \Gamma} \leq C\left\{\frac{\widehat{h}}{\widetilde{h}}\right\}^{\delta}\|\xi\|_{-1 / 2, \Gamma} .
\end{aligned}
$$

Similarly, applying triangle inequality, estimate (3.4), and trace theorem, and then assuming that $\widehat{h} \leq \widetilde{h}$, we obtain

$$
\begin{aligned}
\left\|\widehat{\psi}_{\widehat{h}}\right\|_{1 / 2, \Gamma} & \leq\left\|\psi-\widehat{\psi}_{\widehat{h}}\right\|_{1 / 2, \Gamma}+\|\psi\|_{1 / 2, \Gamma} \\
& \leq C\left\{\begin{array}{c}
\widehat{h} \\
\widetilde{\widetilde{h}}
\end{array}\right\}^{\delta}\|\xi\|_{-1 / 2, \Gamma}+\|z\|_{1, \Omega} \leq C\|\xi\|_{-1 / 2, \Gamma} .
\end{aligned}
$$

On the other hand, it is easy to see that

$$
\langle\xi, \psi\rangle=\left\langle\partial_{\nu} z, z\right\rangle=\|z\|_{1, \Omega}^{2} \geq C\left\|\partial_{\nu} z\right\|_{-1 / 2, \Gamma}^{2}=C\|\xi\|_{-1 / 2, \Gamma}^{2} .
$$

Consequently, employing (3.4), (3.5), and (3.6), we can write

$$
\begin{aligned}
|\xi|_{\widehat{h}} & :=\sup _{0 \neq \psi_{\hat{h}} \in Y_{\widehat{h}}} \frac{\left\langle\xi, \psi_{\widehat{h}}\right\rangle}{\left\|\psi_{\widehat{h}}\right\|_{1 / 2, \Gamma}} \geq \frac{\left|\left\langle\xi, \widehat{\psi}_{\widehat{h}}\right\rangle\right|}{\left\|\widehat{\psi}_{\widehat{h}}\right\|_{1 / 2, \Gamma}} \geq C \frac{\left|\left\langle\xi, \widehat{\psi}_{\hat{h}}\right\rangle\right|}{\|\xi\|_{-1 / 2, \Gamma}} \\
& \geq \frac{|\langle\xi, \psi\rangle|}{\|\xi\|_{-1 / 2, \Gamma}}-\frac{\left|\left\langle\xi, \widehat{\psi}_{\widehat{h}}-\psi\right\rangle\right|}{\|\xi\|_{-1 / 2, \Gamma}} \geq \bar{c}\|\xi\|_{-1 / 2, \Gamma}-\bar{C}\left\{\frac{\widehat{h}}{\widetilde{h}}\right\}^{\delta}\|\xi\|_{-1 / 2, \Gamma},
\end{aligned}
$$

which completes the proof with constants $C_{0}$ and $\tilde{C}$ depending on $\bar{c}, \bar{C}$, and $\delta$.

We now concentrate on the analysis of the reduced coupled LDG-BEM scheme (2.25). We begin with the necessary estimates for the bilinear form $B_{h}$. 
Lemma 3.2. There exist positive constants $c, C$, independent of $h$, such that

$$
\left|B_{h}(w, v)\right| \leq c\|w\|_{h}\|v\|_{h} \quad \forall w, v \in H^{1}\left(\mathcal{T}_{h}\right)
$$

and

$$
B_{h}(v, v) \geq C\|v\|_{h}^{2} \quad \forall v \in H^{1}\left(\mathcal{T}_{h}\right) .
$$

Proof. We refer to Proposition 3.1 in [28] (see also Lemmas 4.1 and 4.2 in [5] for the corresponding nonlinear case). We only recall here, for further use, that

$$
|S(w, \tau)| \leq C|w|_{*}\left\{\sum_{K \in \mathcal{T}_{h}} h_{K}\|\tau\|_{0, \partial K}^{2}\right\}^{1 / 2} \quad \forall(w, \tau) \in H^{1}\left(\mathcal{T}_{h}\right) \times \mathbf{H}^{1}\left(\mathcal{T}_{h}\right),
$$

which, according to a discrete trace inequality and a simple inverse estimate, yields

$$
\left\|\mathbf{S}_{h}(w)\right\|_{0, \Omega} \leq C|w|_{*} \quad \forall w \in H^{1}\left(\mathcal{T}_{h}\right) .
$$

The boundedness of the operator $\mathbf{g}_{\widehat{h}}$ with respect to the seminorm $|\cdot|_{\widehat{h}}$ is now established.

Lemma 3.3. There exists a positive constant $C$, independent of the meshsizes, such that for all $\xi \in H^{-1 / 2}(\Gamma)$

$$
\left\|\mathbf{g}_{\widehat{h}}(\xi)\right\|:=\left\|\mathbf{g}_{\widehat{h}}^{1}(\xi)\right\|_{1 / 2, \Gamma}+\left\|\mathbf{g}_{\widehat{h}}^{2}(\xi)\right\|_{-1 / 2, \Gamma} \leq C|\xi|_{\widehat{h}} .
$$

Proof. Since $\mathbf{D}$ is elliptic on $H_{0}^{1 / 2}(\Gamma) \times H_{0}^{-1 / 2}(\Gamma)$, there exists $C>0$ such that

$$
C\|(\psi, \mu)\|^{2} \leq \mathbf{D}((\psi, \mu),(\psi, \mu)) \quad \forall(\psi, \mu) \in H_{0}^{1 / 2}(\Gamma) \times H_{0}^{-1 / 2}(\Gamma) .
$$

In particular, given $\xi \in H^{-1 / 2}(\Gamma)$, it follows that

$$
C\left\|\mathbf{g}_{\widehat{h}}(\xi)\right\|^{2} \leq \mathbf{D}\left(\mathbf{g}_{\widehat{h}}(\xi), \mathbf{g}_{\widehat{h}}(\xi)\right)=\left\langle\xi, \mathbf{g}_{\widehat{h}}^{1}(\xi)\right\rangle \leq|\xi|_{\widehat{h}}\left\|\mathbf{g}_{\widehat{h}}^{1}(\xi)\right\|_{1 / 2, \Gamma} \leq|\xi|_{\widehat{h}}\left\|\mathbf{g}_{\widehat{h}}(\xi)\right\|,
$$

which yields the required estimate.

A symmetry property and further estimates for the first component of $\mathbf{g}_{\widehat{h}}$ are shown next.

Lemma 3.4. There holds

$$
\left\langle\xi, \mathbf{g}_{\widehat{h}}^{1}(\mu)\right\rangle=\left\langle\mu, \mathbf{g}_{\widehat{h}}^{1}(\xi)\right\rangle \quad \forall \xi, \mu \in H^{-1 / 2}(\Gamma) .
$$

In addition, there exist positive constants $c, C$, independent of the meshsizes, such that

$$
\left|\left\langle\xi, \mathbf{g}_{\widehat{h}}^{1}(\mu)\right\rangle\right| \leq c|\xi|_{\widehat{h}}|\mu|_{\widehat{h}} \quad \forall \xi, \mu \in H^{-1 / 2}(\Gamma)
$$

and

$$
\left\langle\xi, \mathbf{g}_{\widehat{h}}^{1}(\xi)\right\rangle \geq C|\xi|_{\widehat{h}}^{2} \quad \forall \xi \in H_{0}^{-1 / 2}(\Gamma) .
$$

Proof. Let $\xi, \mu \in H^{-1 / 2}(\Gamma)$. Using the definition of $\mathbf{g}_{\hat{h}}$ (cf. (2.24) $)$ and the symmetry of $\overline{\mathbf{D}}$, we obtain

$$
\left\langle\xi, \mathbf{g}_{\widehat{h}}^{1}(\mu)\right\rangle=\overline{\mathbf{D}}\left(\mathbf{g}_{\widehat{h}}(\xi), \mathbf{g}_{\widehat{h}}(\mu)\right)=\overline{\mathbf{D}}\left(\mathbf{g}_{\widehat{h}}(\mu), \mathbf{g}_{\widehat{h}}(\xi)\right)=\left\langle\mu, \mathbf{g}_{\widehat{h}}^{1}(\xi)\right\rangle,
$$

which proves (3.11). Now, noting that $\mathbf{g}_{\widehat{h}}^{1}(\mu) \in Y_{\widehat{h}}^{0} \subseteq Y_{\widehat{h}}$ and applying the definition of $|\cdot|_{\widehat{h}}$ and Lemma 3.3, we can write

$$
\left|\left\langle\xi, \mathbf{g}_{\widehat{h}}^{1}(\mu)\right\rangle\right| \leq|\xi|_{\widehat{h}}\left\|\mathbf{g}_{\widehat{h}}^{1}(\mu)\right\|_{1 / 2, \Gamma} \leq|\xi|_{\widehat{h}}\left\|\mathbf{g}_{\widehat{h}}(\mu)\right\| \leq c|\xi|_{\widehat{h}}|\mu|_{\widehat{h}},
$$

which is 3.12). 
On the other hand, we note that each $\psi_{\widehat{h}} \in Y_{\widehat{h}}$ can be decomposed as $\psi_{\widehat{h}}=\psi_{\widehat{h}}^{0}+c$, with $\psi_{\widehat{h}}^{0}:=\left(\psi_{\widehat{h}}-\frac{1}{|\Gamma|} \int_{\Gamma} \psi_{\widehat{h}}\right) \in Y_{\widehat{h}}^{0}$ and $c:=\frac{1}{|\Gamma|} \int_{\Gamma} \psi_{\widehat{h}} \in P_{0}(\Gamma)$. This decomposition is stable, and there exists $C>0$ such that $\left\|\psi_{\widehat{h}}^{0}\right\|_{1 / 2, \Gamma} \leq C\left\|\psi_{\widehat{h}}\right\|_{1 / 2, \Gamma}$ for all $\psi_{\widehat{h}} \in Y_{\widehat{h}}$. In addition, given $\xi \in H_{0}^{-1 / 2}(\Gamma)$, it is clear that $\left\langle\xi, \psi_{\widehat{h}}\right\rangle=\left\langle\xi, \psi_{\widehat{h}}^{0}\right\rangle$ for all $\psi_{\widehat{h}} \in Y_{\widehat{h}}$.

Therefore, applying the definition of $\mathbf{g}_{\widehat{h}}$ (cf. (2.23) ), and the boundedness and ellipticity of $\mathbf{D}$, it follows that

$$
\begin{aligned}
& |\xi|_{\widehat{h}}=\sup _{0 \neq \psi_{\hat{h}} \in Y_{\widehat{h}}} \frac{\left\langle\xi, \psi_{\widehat{h}}\right\rangle}{\left\|\psi_{\widehat{h}}\right\|_{1 / 2, \Gamma}} \leq C \sup _{0 \neq \psi_{\hat{h}} \in Y_{\widehat{h}}^{0}} \frac{\left\langle\xi, \psi_{\widehat{h}}\right\rangle}{\left\|\psi_{\widehat{h}}\right\|_{1 / 2, \Gamma}} \\
& =C \sup _{0 \neq \psi_{\widehat{h}} \in Y_{\widehat{h}}^{0}} \frac{\mathbf{D}\left(\mathbf{g}_{\widehat{h}}(\xi),\left(\psi_{\widehat{h}}, 0\right)\right)}{\left\|\psi_{\widehat{h}}\right\|_{1 / 2, \Gamma}} \leq C\left\|\mathbf{g}_{\widehat{h}}(\xi)\right\| \\
& \leq \bar{C} \mathbf{D}\left(\mathbf{g}_{\widehat{h}}(\xi), \mathbf{g}_{\widehat{h}}(\xi)\right)^{1 / 2}=\bar{C}\left\langle\xi, \mathbf{g}_{\widehat{h}}^{1}(\xi)\right\rangle^{1 / 2},
\end{aligned}
$$

which yields (3.13).

We now define the bilinear form $\mathbf{A}_{h, \widehat{h}}:\left(H^{1}\left(\mathcal{T}_{h}\right) \times L^{2}(\Gamma)\right) \times\left(H^{1}\left(\mathcal{T}_{h}\right) \times L^{2}(\Gamma)\right) \rightarrow$ $\mathbb{R}$ by

$$
\mathbf{A}_{h, \widehat{h}}((w, \mu),(v, \xi)):=B_{h}(w, v)-\langle\mu, v\rangle+\langle\xi, w\rangle+\left\langle\xi, \mathbf{g}_{\widehat{h}}^{1}(\mu)\right\rangle,
$$

for all $(w, \mu),(v, \xi) \in H^{1}\left(\mathcal{T}_{h}\right) \times L^{2}(\Gamma)$. Then, the main result of this section is established as follows.

Theorem 3.1. The coupled LDG-BEM scheme (2.19), and hence the equivalent formulation (2.25), is uniquely solvable, and there holds the stability estimate:

$$
\left\|u_{h}\right\|_{h}+\left|\lambda_{\tilde{h}}\right|_{\widehat{h}}+\left\|\sigma_{h}\right\|_{0, \Omega}+\left\|\varphi_{\widehat{h}}\right\|_{1 / 2, \Gamma}+\left\|\gamma_{\widehat{h}}\right\|_{-1 / 2, \Gamma} \leq C\|f\|_{0, \Omega} .
$$

Proof. We first observe, by virtue of Lemma 2.3, that the unique solvability of (2.19) is equivalent to that of problem (2.25), which can be reformulated as: find $\left(u_{h}, \lambda_{\tilde{h}}\right) \in V_{h} \times X_{\widetilde{h}}^{0}$ such that

$$
\mathbf{A}_{h, \widehat{h}}\left(\left(u_{h}, \lambda_{\tilde{h}}\right),(v, \xi)\right)=\int_{\Omega} f v \quad \forall(v, \xi) \in V_{h} \times X_{\widetilde{h}}^{0} .
$$

It follows from (3.8) and (3.13) that $\mathbf{A}_{h, \widehat{h}}$ is elliptic on the product space $V_{h} \times X_{\widetilde{h}}^{0}$ endowed with the norm $\left(\|\cdot\|_{h}^{2}+|\cdot|_{\hat{h}}^{2}\right)^{1 / 2}$, and hence the discrete scheme (3.15) is uniquely solvable. Then, applying the estimate $\|v\|_{0, \Omega} \leq C\|v\|_{h} \forall v \in V_{h}$ (see [1]) and the ellipticity of $\mathbf{A}_{h, \widehat{h}}$ again, we deduce that

$$
\left\|u_{h}\right\|_{h}+\left|\lambda_{\tilde{h}}\right|_{\widehat{h}} \leq C \sup _{\substack{0 \neq v_{h} \in V_{h} \\\left\|v_{h}\right\|_{h}}}\left|\int_{\Omega} f v_{h}\right| \leq C\|f\|_{0, \Omega} .
$$

The remaining unknowns are written in terms of $u_{h}$ and $\lambda_{\widetilde{h}}$ (see Lemma 2.3), and therefore they can be bounded using (3.10) and Lemma 3.3. We omit further details. 


\section{A PRIORI ERROR ANALYSIS}

In order to perform the a priori error analysis for the solution of our discrete scheme (2.19), we need further notation. We let

$$
\varepsilon(\widehat{h}, \widetilde{h}):=\sup _{0 \neq \xi_{\tilde{h}} \in X_{\tilde{h}}} \frac{\left\|\xi_{\tilde{h}}\right\|_{0}}{\left|\xi_{\tilde{h}}\right|_{\widehat{h}}}
$$

define the boundary function $h_{\Gamma} \in P_{0}\left(\Gamma_{h}\right)$ by $\left.h_{\Gamma}\right|_{e}:=h_{e} \forall e \in \mathcal{E}_{h}^{\Gamma}$, and introduce two new norms,

$$
\|v\|_{h, \widehat{h}, \widetilde{h}}^{2}:=\|v\|_{h}^{2}+\varepsilon(\widehat{h}, \widetilde{h})^{2}\|v\|_{0, \Gamma}^{2} \quad \forall v \in H^{1}\left(\mathcal{T}_{h}\right)
$$

and

$$
\|\xi\|_{h, \widehat{h}}^{2}:=|\xi|_{\widehat{h}}^{2}+\left\|h_{\Gamma}^{-1 / 2} \xi\right\|_{0}^{2} \quad \forall \xi \in L^{2}(\Gamma),
$$

where the term $\|v\|_{0, \Gamma}$ refers to the $L^{2}(\Gamma)$ norm of the piecewisely defined trace of $v \in H^{1}\left(\mathcal{T}_{h}\right)$.

Assuming that $|\cdot|_{\widehat{h}}$ is equivalent to $\|\cdot\|_{-1 / 2, \Gamma}$ on $X_{\widetilde{h}}$, which is guaranteed by Lemma 3.1, and applying the inverse inequality (3.2), it follows that $\varepsilon(\widehat{h}, \widetilde{h}) \leq$ $C \widetilde{h}^{-1 / 2}$. This upper bound will be used below in Lemma 4.4 .

We now prove a boundedness property of $\mathbf{A}_{h, \widehat{h}}$ with respect to the mesh-dependent norms.

Lemma 4.1. There exists $C>0$ such that

$$
\left|\mathbf{A}_{h, \widehat{h}}((w, \mu),(v, \xi))\right| \leq C\left(\|w\|_{h, \widehat{h}, \tilde{h}}+\|\mu\|_{h, \widehat{h}}\right)\left(\|v\|_{h}+|\xi|_{\widehat{h}}\right),
$$

for all $(w, \mu) \in H^{1}\left(\mathcal{T}_{h}\right) \times L^{2}(\Gamma)$ and $(v, \xi) \in V_{h} \times X_{\widetilde{h}}$.

Proof. It is easy to see, according to the definitions of $\|\cdot\|_{h, \widehat{h}, \widetilde{h}}$ and $\varepsilon(\widehat{h}, \widetilde{h})$, that

$$
|\langle w, \xi\rangle| \leq \varepsilon(\widehat{h}, \widetilde{h})\|w\|_{0, \Gamma}|\xi|_{\widehat{h}} \leq\|w\|_{h, \widehat{h}, \widetilde{h}}|\xi|_{\widehat{h}} \quad \forall w \in H^{1}\left(\mathcal{T}_{h}\right), \quad \forall \xi \in X_{\widetilde{h}} .
$$

On the other hand, by using a discrete trace inequality (see equation (2.4) in [1]), a local inverse inequality, and the estimate $\|v\|_{0, \Omega} \leq C\|v\|_{h} \forall v \in V_{h}$ (see [1]), we find that for arbitrary $v \in V_{h}$

$$
\begin{aligned}
\left\|h_{\Gamma}^{1 / 2} v\right\|_{0, \Gamma}^{2} & =\sum_{e \in \mathcal{E}_{h}^{\Gamma}} h_{e}\|v\|_{0, e}^{2} \leq C \sum_{K \in \mathcal{T}_{h, \Gamma}}\left\{\|v\|_{0, K}^{2}+h_{K}^{2}\left|v_{h}\right|_{1, K}^{2}\right\} \\
& \leq C \sum_{K \in \mathcal{T}_{h, \Gamma}}\|v\|_{0, K}^{2} \leq C\|v\|_{0, \Omega}^{2} \leq C\|v\|_{h}^{2},
\end{aligned}
$$

where $\mathcal{T}_{h, \Gamma}$ denotes the triangles of $\mathcal{T}_{h}$ with sides on $\Gamma$. It follows, using CauchySchwarz's inequality in $L^{2}(\Gamma)$, that

$$
|\langle\mu, v\rangle| \leq\left\|h_{\Gamma}^{-1 / 2} \mu\right\|_{0, \Gamma}\left\|h_{\Gamma}^{1 / 2} v\right\|_{0, \Gamma} \leq C\|\mu\|_{h, \widehat{h}}\|v\|_{h} \quad \forall \mu \in L^{2}(\Gamma), \quad \forall v \in V_{h} .
$$

The result is then a straightforward consequence of (3.7), (3.12), (4.1), and (4.2).

The following theorem provides a Strang-type error estimate for the solution of our discrete scheme (2.19). 
Theorem 4.1. There exists $C>0$, independent of the meshsizes, such that

$$
\begin{array}{r}
\left\|u-u_{h}\right\|_{h}+\left|\lambda-\lambda_{\widehat{h}}\right|_{\widehat{h}}+\left\|\sigma-\sigma_{h}\right\|_{0, \Omega}+\left\|\varphi-\varphi_{\widehat{h}}\right\|_{1 / 2, \Gamma}+\left\|\gamma-\gamma_{\widehat{h}}\right\|_{-1 / 2, \Gamma} \\
\leq C\left\{R_{h, \widehat{h}, \widetilde{h}}(u)+\inf _{v_{h} \in V_{h}}\left\|u-v_{h}\right\|_{h, \widehat{h}, \widetilde{h}}+\inf _{\xi_{\tilde{h}} \in X_{\widehat{h}}^{0}}\left\|\lambda-\xi_{\widetilde{h}}\right\|_{h, \widehat{h}}\right. \\
\left.+\inf _{\psi_{\hat{h}} \in Y_{\hat{h}}^{0}}\left\|\varphi-\psi_{\widehat{h}}\right\|_{1 / 2, \Gamma}+\inf _{\mu_{\widehat{h}} \in Z_{\hat{h}}^{0}}\left\|\gamma-\mu_{\widehat{h}}\right\|_{-1 / 2, \Gamma}\right\},
\end{array}
$$

where $R_{h, \widehat{h}, \widetilde{h}}(u)$ is a consistency term given by

$$
\mathcal{R}_{h, \widehat{h}, \widetilde{h}}(u):=\sup _{(0,0) \neq\left(v_{h}, \xi_{\tilde{h}}\right) \in V_{h} \times X_{\widetilde{h}}^{0}} \frac{\mathbf{A}_{h, \widehat{h}}\left(\left(u, \partial_{\nu} u\right),\left(v_{h}, \xi_{\widetilde{h}}\right)\right)-\int_{\Omega} f v_{h}}{\left\|v_{h}\right\|_{h}+\left|\xi_{\widetilde{h}}\right|_{\widehat{h}}} .
$$

Proof. Applying the ellipticity of $\mathbf{A}_{h, \widehat{h}}$, adding and subtracting $\left(u, \partial_{\nu} u\right)$ in the first component of $\mathbf{A}_{h, \widehat{h}}$, and then using (3.15) and Lemma 4.1. we deduce that for arbitrary $\left(v_{h}, \xi_{\tilde{h}}\right) \in V_{h} \times X_{\widetilde{h}}^{0}$ there holds

$$
\left\|u_{h}-v_{h}\right\|_{h}+\left|\lambda_{\widetilde{h}}-\xi_{\tilde{h}}\right|_{\widehat{h}} \leq C\left\{R_{h, \widehat{h}, \widetilde{h}}(u)+\left\|u-v_{h}\right\|_{h, \widehat{h}, \widetilde{h}}+\left\|\lambda-\xi_{\widetilde{h}}\right\|_{h, \widehat{h}}\right\},
$$

which, employing triangle inequality, yields

$$
\begin{aligned}
& \left\|u-u_{h}\right\|_{h}+\left|\lambda-\lambda_{\tilde{h}}\right|_{\widehat{h}} \\
& \leq C\left\{R_{h, \widehat{h}, \tilde{h}}(u)+\inf _{v_{h} \in V_{h}}\left\|u-v_{h}\right\|_{h, \widehat{h}, \tilde{h}}+\inf _{\xi_{\tilde{h}} \in X_{\tilde{h}}^{0}}\left\|\lambda-\xi_{\tilde{h}}\right\|_{h, \widehat{h}}\right\} .
\end{aligned}
$$

This bounds the first and second term of the error. Now, since $\sigma=\nabla u=$ $\nabla u-\mathbf{S}_{h}(u)$ and $\sigma_{h}=\nabla_{h} u_{h}-\mathbf{S}_{h}\left(u_{h}\right)$ (cf. Lemma 2.2), we easily obtain, using (3.10), that

$$
\left\|\sigma-\sigma_{h}\right\|_{0, \Omega} \leq C\left\|u-u_{h}\right\|_{h},
$$

which, thanks to (4.5), bounds the third term.

Next, we note that $\left(\varphi_{\widehat{h}}, \gamma_{\widehat{h}}\right):=\mathbf{g}_{\widehat{h}}\left(-\lambda_{\widetilde{h}}\right)$ is a nonconforming Galerkin approximation with a modified right-hand side $\left(\lambda_{\tilde{h}}\right.$ instead of $\left.\lambda\right)$ of the solution $(\varphi, \gamma)$ of the elliptic problem (2.5). Therefore, by the first Strang Lemma we have

$$
\begin{aligned}
& \left\|\varphi-\varphi_{\widehat{h}}\right\|_{1 / 2, \Gamma}+\left\|\gamma-\gamma_{\widehat{h}}\right\|_{-1 / 2, \Gamma} \\
& \leq C\left\{\inf _{\psi_{\hat{h}} \in Y_{\hat{h}}^{0}}\left\|\varphi-\psi_{\widehat{h}}\right\|_{1 / 2, \Gamma}+\inf _{\mu_{\hat{h}} \in Z_{\hat{h}}^{0}}\left\|\gamma-\mu_{\hat{h}}\right\|_{-1 / 2, \Gamma}+\left|\lambda-\lambda_{\tilde{h}}\right|_{\widehat{h}}\right\},
\end{aligned}
$$

since the seminorm $|\cdot|_{\widehat{h}}$ measures the approximation error in the right-hand side. The above estimate together with (4.5) and (4.6) complete the proof.

The Strang-type estimate (4.3) will be used to derive the explicit a priori error estimate. To this end, we now observe, according to the $\mathcal{C}^{\infty}$-regularity of the harmonic function $u$ in the exterior of the support of $f$, that $\varphi=\left.u\right|_{\Gamma} \in H^{s}(\Gamma)$ and $\lambda=\gamma=\partial_{\nu} u \in H^{s}(\Gamma)$ for any $s \in \mathbb{R}$. In addition, a usual regularity result on Lipschitz domains guarantees that $u \in H^{1+\delta}(\Omega)$, for some $\delta>1 / 2$. 
On the other hand, the following lemma establishing well-known approximation properties of piecewise polynomials (see, e.g., [9], 20]) is needed for the local estimates.

Lemma 4.2. Given a nonnegative integer $k$, let $\Pi_{K}^{k}: L^{2}(K) \rightarrow P_{k}(K)$ be the linear and bounded operator given by the $L^{2}(K)$-orthogonal projection, which satisfies $\Pi_{K}^{k}(p)=p$ for all $p \in P_{k}(K)$, and let $\mathbf{I}$ be a generic identity operator. Then there exists $C>0$, independent of the meshsizes, such that for each $s, t$ satisfying $0 \leq s \leq k+1$ and $0 \leq s<t$, there holds

$$
\left|\left(\mathbf{I}-\Pi_{K}^{k}\right)(w)\right|_{s, K} \leq C h_{K}^{\min \{t, k+1\}-s}\|w\|_{t, K} \quad \forall w \in H^{t}(K),
$$

and for each $t>1 / 2$ there holds

$$
\left\|\left(\mathbf{I}-\Pi_{K}^{k}\right)(w)\right\|_{0, \partial K} \leq C h_{K}^{\min \{t, k+1\}-1 / 2}\|w\|_{t, K} \quad \forall w \in H^{t}(K) .
$$

In order to now estimate the consistency term $R_{h, \widehat{h}, \widetilde{h}}(u)$, we recall from (2.7) that the orthogonal projector $\Pi_{\Sigma_{h}}$ reduces locally to $\Pi_{K}^{r}$ on each $K \in \mathcal{T}_{h}$, where $r=m$ or $r=m-1$, with $m \in \mathbb{N}$.

Lemma 4.3. There exists $C>0$, independent of the meshsizes, such that

$$
\begin{aligned}
R_{h, \widehat{h}, \widehat{h}}(u) \leq C\{ & \sum_{K \in \mathcal{T}_{h}} h_{K}^{2 \min \{\delta, r+1\}}\|u\|_{1+\delta, K}^{2} \\
& \left.+\inf _{\psi_{\hat{h}} \in Y_{\widehat{h}}^{0}}\left\|\varphi-\psi_{\widehat{h}}\right\|_{1 / 2, \Gamma}^{2}+\inf _{\mu_{\widehat{h}} \in Z_{\hat{h}}^{0}}\left\|\gamma-\mu_{\widehat{h}}\right\|_{-1 / 2, \Gamma}^{2}\right\}^{1 / 2} .
\end{aligned}
$$

Proof. It follows easily from the definition of $\mathbf{A}_{h, \widehat{h}}$ (cf. (3.14) ) and Lemma 2.1 that

$$
\mathbf{A}_{h, \widehat{h}}\left(\left(u, \partial_{\nu} u\right),\left(v_{h}, \xi_{\widetilde{h}}\right)\right)=\int_{\Omega} f v_{h}+S\left(v_{h}, \nabla u-\Pi_{\boldsymbol{\Sigma}_{h}}(\nabla u)\right)+\left\langle\xi_{\widetilde{h}}, \varphi+\mathbf{g}_{\widehat{h}}^{1}(\lambda)\right\rangle
$$

for all $\left(v_{h}, \xi_{\widetilde{h}}\right) \in V_{h} \times X_{\widetilde{h}}^{0}$. Then, replacing the above in (4.4), using the first estimate in (3.9), and employing the equivalence of $|\cdot|_{\widehat{h}}$ and $\|\cdot\|_{-1 / 2, \Gamma}$ in $X_{\widetilde{h}}$, we find

$$
R_{h, \widehat{h}, \widetilde{h}}(u) \leq C\left\{\sum_{K \in \mathcal{T}_{h}} h_{K}\left\|\nabla u-\Pi_{\boldsymbol{\Sigma}_{h}}(\nabla u)\right\|_{0, \partial K}^{2}\right\}^{1 / 2}+C\left\|\varphi+\mathbf{g}_{\widehat{h}}^{1}(\lambda)\right\|_{1 / 2, \Gamma} .
$$

Next, applying (4.8) to $w=\left.\nabla u\right|_{K} \in H^{\delta}(K)$, we deduce that

$$
\begin{gathered}
\sum_{K \in \mathcal{T}_{h}} h_{K}\left\|\nabla u-\Pi_{\boldsymbol{\Sigma}_{h}}(\nabla u)\right\|_{0, \partial K}^{2}=\sum_{K \in \mathcal{T}_{h}} h_{K}\left\|\left(\mathbf{I}-\Pi_{K}^{r}\right)(\nabla u)\right\|_{0, \partial K}^{2} \\
\leq C \sum_{K \in \mathcal{T}_{h}} h_{K}^{2 \min \{\delta, r+1\}}\|u\|_{1+\delta, K}^{2} .
\end{gathered}
$$

Finally, since $\mathbf{g}_{\widehat{h}}(-\lambda):=\left(\mathbf{g}_{\widehat{h}}^{1}(-\lambda), \mathbf{g}_{\widehat{h}}^{2}(-\lambda)\right)$ is the Galerkin approximation of the solution $(\varphi, \gamma)$ of the elliptic problem (2.5), we obtain from the Cea lemma that

$$
\left\|\varphi+\mathbf{g}_{\widehat{h}}^{1}(\lambda)\right\|_{1 / 2, \Gamma} \leq C\left\{\inf _{\psi_{\hat{h}} \in Y_{\widehat{h}}^{0}}\left\|\varphi-\psi_{\widehat{h}}\right\|_{1 / 2, \Gamma}+\inf _{\mu_{\hat{h}} \in Z_{\hat{h}}^{0}}\left\|\gamma-\mu_{\widehat{h}}\right\|_{-1 / 2, \Gamma}\right\},
$$

which completes the proof. 
We now let $\Pi_{V_{h}}$ be the $L^{2}(\Omega)$-projection onto $V_{h}$ and observe from (2.7) that $\Pi_{V_{h}}$ reduces locally to $\Pi_{K}^{m}$ on each $K \in \mathcal{T}_{h}$, with $m \in \mathbb{N}$. Then, we have the following result.

Lemma 4.4. Assume that $h=O(\widetilde{h})$. Then, there exists $C>0$, independent of the meshsizes, such that

$$
\inf _{v_{h} \in V_{h}}\left\|u-v_{h}\right\|_{h, \widehat{h}, \widetilde{h}} \leq C\left\{\sum_{K \in \mathcal{T}_{h}} h_{K}^{2 \min \{\delta, m\}}\|u\|_{1+\delta, K}^{2}\right\}^{1 / 2} .
$$

Proof. We clearly have

$\inf _{v_{h} \in V_{h}}\left\|u-v_{h}\right\|_{h, \widehat{h}, \tilde{h}}^{2} \leq\left\|u-\Pi_{V_{h}} u\right\|_{h, \widehat{h}, \widetilde{h}}^{2}=\left\|u-\Pi_{V_{h}} u\right\|_{h}^{2}+\varepsilon(\widehat{h}, \widetilde{h})^{2}\left\|u-\Pi_{V_{h}} u\right\|_{0, \Gamma}^{2}$.

Then, the upper bound for $\|\cdot\|_{h}$, given by Lemma 5.3 in [5], establishes that

$$
\left\|u-\Pi_{V_{h}} u\right\|_{h}^{2} \leq C \sum_{K \in \mathcal{T}_{h}}\left\{\left|\left(\mathbf{I}-\Pi_{K}^{m}\right)(u)\right|_{1, K}^{2}+h_{K}^{-1}\left\|\left(\mathbf{I}-\Pi_{K}^{m}\right)(u)\right\|_{0, \partial K}^{2}\right\},
$$

which, applying (4.7) and (4.8) with $s=1$ and $t=1+\delta$, yields

$$
\left\|u-\Pi_{V_{h}} u\right\|_{h}^{2} \leq C \sum_{K \in \mathcal{T}_{h}} h_{K}^{2 \min \{\delta, m\}}\|u\|_{1+\delta, K}^{2} .
$$

On the other hand, using a discrete trace inequality (see equation (2.4) in [1]), we can write

$$
\left\|u-\Pi_{V_{h}} u\right\|_{0, \Gamma}^{2} \leq C \sum_{K \in \mathcal{T}_{h, \Gamma}}\left\{h_{K}^{-1}\left\|\left(\mathbf{I}-\Pi_{K}^{m}\right)(u)\right\|_{0, K}^{2}+h_{K}\left|\left(\mathbf{I}-\Pi_{K}^{m}\right)(u)\right|_{1, K}^{2}\right\},
$$

which, now applying (4.7) with $s=0$ and $s=1$, and using that $\varepsilon(\widehat{h}, \widetilde{h})^{2} \leq C \widetilde{h}^{-1}$ and that $h=O(\widetilde{h})$, gives

$$
\varepsilon(\widehat{h}, \widetilde{h})^{2}\left\|u-\Pi_{V_{h}} u\right\|_{0, \Gamma}^{2} \leq C \sum_{K \in \mathcal{T}_{h, \Gamma}} h_{K}^{2 \min \{\delta, m\}}\|u\|_{1+\delta, K}^{2} .
$$

Thus, the required upper bound follows from (4.9), (4.10), and (4.11).

We now estimate the distance to $X_{\widetilde{h}}^{0}$ with respect to the mesh-dependent norm $\|\cdot\|_{h, \widehat{h}}$.

Lemma 4.5. Assume that $h=O(\widetilde{h})$. Then, for each $t>1 / 2$ there exists $C>0$, independent of the meshsizes, such that

$$
\inf _{\xi_{\tilde{h}} \in X_{\tilde{h}}^{0}}\left\|\xi-\xi_{\widetilde{h}}\right\|_{h, \widehat{h}} \leq C \widetilde{h}^{\min \{t-1 / 2, \widetilde{k}\}}\|\xi\|_{t, \Gamma} \quad \forall \xi \in H^{t}(\Gamma) \cap H_{0}^{-1 / 2}(\Gamma) .
$$

Proof. Let $\xi \in H^{t}(\Gamma) \cap H_{0}^{-1 / 2}(\Gamma)$ with $t>1 / 2$. Then, there exists $w \in H^{t+1 / 2}(\Omega)$ such that $w=\xi$ on $\Gamma$ and $\|w\|_{t+1 / 2, \Omega} \leq C\|\xi\|_{t, \Gamma}$. Next, we observe from the definition of $\|\cdot\|_{h, \widehat{h}}$ that $\|\cdot\|_{h, \widehat{h}} \leq C h^{-1 / 2}\|\cdot\|_{0, \Gamma}$ on $L^{2}(\Gamma)$, and hence, using that $h=O(\widetilde{h})$, we find that

$$
\inf _{\xi_{\tilde{h}} \in X_{\tilde{h}}^{0}}\left\|\xi-\xi_{\widetilde{h}}\right\|_{h, \widehat{h}} \leq C \widetilde{h}^{-1 / 2} \inf _{\xi_{\tilde{h}} \in X_{\tilde{h}}^{0}}\left\|\xi-\xi_{\widetilde{h}}\right\|_{0, \Gamma} .
$$


Now, to each $\widetilde{e} \in \mathcal{E}_{\widetilde{h}}^{\Gamma}$ we associate a regular triangle $\widetilde{K} \subseteq \Omega$ such that $\partial \widetilde{K} \cap \Gamma=\widetilde{e}$, and set $\mathcal{T}_{\widetilde{h}, \Gamma}$ for the list of all of them. Then, we define the function $\mu_{\widetilde{h}} \in X_{\widetilde{h}}$ by $\left.\mu_{\widetilde{h}}\right|_{\tilde{e}}=\left.\Pi_{\widetilde{K}}^{\widetilde{k}}(w)\right|_{\widetilde{e}}$ for all $\widetilde{e} \in \mathcal{E}_{\widetilde{h}}^{\Gamma}$, and denote by $\mu_{\widetilde{h}}^{0}$ its component in $X_{\widetilde{h}}^{0}$. It follows easily that

$$
\begin{aligned}
\left\|\xi-\mu_{\widetilde{h}}^{0}\right\|_{0, \Gamma}^{2} & \leq\left\|\xi-\mu_{\tilde{h}}\right\|_{0, \Gamma}^{2}=\left\|w-\mu_{\widetilde{h}}\right\|_{0, \Gamma}^{2} \\
& =\sum_{\widetilde{e} \in \mathcal{E}_{\tilde{h}}^{\Gamma}}\left\|w-\mu_{\tilde{h}}\right\|_{0, \widetilde{e}}^{2} \leq \sum_{\widetilde{K} \in \mathcal{T}_{\widetilde{h}, \Gamma}\left\|w-\Pi_{\widetilde{K}}^{\widetilde{K}}(w)\right\|_{0, \partial \widetilde{K}}^{2}},
\end{aligned}
$$

which, applying (4.8) to $k=\widetilde{k}$ and $w \in H^{t+1 / 2}(\Omega)$, yields

$$
\left\|\xi-\mu_{\widetilde{h}}^{0}\right\|_{0, \Gamma}^{2} \leq C \sum_{\widetilde{K} \in \mathcal{T}_{\widetilde{h}, \Gamma}} h_{\widetilde{K}}^{2 \widetilde{m i n}\{t, \widetilde{k}+1 / 2\}}\|w\|_{t+1 / 2, \widetilde{K}}^{2} \leq C \widetilde{h}^{2 \min \{t, \widetilde{k}+1 / 2\}}\|\xi\|_{t, \Gamma}^{2},
$$

and hence

$$
\inf _{\xi_{\tilde{h}} \in X_{\tilde{h}}^{0}}\left\|\xi-\xi_{\widetilde{h}}\right\|_{0, \Gamma}^{2} \leq C \widetilde{h}^{2 \min \{t, \widetilde{k}+1 / 2\}}\|\xi\|_{t, \Gamma}^{2}
$$

In this way, (4.12) and (4.13) complete the proof.

We now recall general approximation properties of the spaces $Y_{\widehat{h}}^{0}$ and $Z_{\widehat{h}}^{0}$. Note that (3.3) is a particular case of (4.14) with $Y_{\widehat{h}}$ instead of $Y_{\widehat{h}}^{0}$.

$\left(\operatorname{AP}\left(Y_{\widehat{h}}^{0}\right)\right)$ For each $s>1 / 2$ there exists $C>0$, independent of $\widehat{h}$, such that

$$
\inf _{\psi_{\hat{h}} \in Y_{\widehat{h}}^{0}}\left\|\psi-\psi_{\widehat{h}}\right\|_{1 / 2, \Gamma} \leq C \widehat{h}^{\min \{s, \widehat{k}+1\}-1 / 2}\|\psi\|_{s, \Gamma} \quad \forall \psi \in H^{s}(\Omega) \cap H_{0}^{1 / 2}(\Gamma) .
$$

$\left(\operatorname{AP}\left(Z_{\widehat{h}}^{0}\right)\right)$ For each $\widehat{t}>-1 / 2$ there exists $C>0$, independent of $\widehat{h}$, such

$$
\inf _{\mu_{\hat{h}} \in Z_{\widehat{h}}^{0}}\left\|\mu-\mu_{\widehat{h}}\right\|_{-1 / 2, \Gamma} \leq C \widehat{h}^{\min \{\hat{t}, \widehat{k}\}+1 / 2}\|\mu\|_{\widehat{t}, \Gamma} \quad \forall \mu \in H^{\widehat{t}}(\Omega) \cap H_{0}^{-1 / 2}(\Gamma) .
$$

Consequently, we summarize the a priori error estimate of our coupled LDGBEM scheme (2.19) in the following theorem.

Theorem 4.2. Assume that $h=O(\widetilde{h})$, that the partition $\Gamma_{\widetilde{h}}$ is uniformly regular, and that $\widehat{h} \leq C_{0} \widetilde{h}$, where $C_{0}>0$ is the constant given by Lemma 3.1. Also, let $r$, $m, \widetilde{k}, \widehat{k}$, and $\widehat{k}-1$ be the polynomial degrees defining locally the subspaces $\boldsymbol{\Sigma}_{h}, V_{h}$, $X_{\widetilde{h}}^{0}, Y_{\widehat{h}}^{0}$, and $Z_{\widehat{h}}^{0}$ (cf. (2.7), (2.10), (2.16)), respectively, and let $\left(\sigma_{h}, u_{h}, \lambda_{\widetilde{h}}, \varphi_{\widehat{h}}, \gamma_{\widehat{h}}\right) \in$ $\Sigma_{h} \times V_{h} \times X_{\widetilde{h}}^{0} \times Y_{\widehat{h}}^{0} \times Z_{\widehat{h}}^{0}$ be the unique solution of our coupled formulation (2.19). In addition, let $\delta>1 / 2$ be such that the exact solution $u$ of (1.2)-(1.3) belongs to 
$H^{1+\delta}(\Omega)$. Then there exists $C>0$, independent of the meshsizes, such that $(4.16)$

$$
\begin{array}{r}
\left\|u-u_{h}\right\|_{h}+\left|\lambda-\lambda_{\widetilde{h}}\right|_{\widehat{h}}+\left\|\sigma-\sigma_{h}\right\|_{0, \Omega}+\left\|\varphi-\varphi_{\widehat{h}}\right\|_{1 / 2, \Gamma}+\left\|\gamma-\gamma_{\widehat{h}}\right\|_{-1 / 2, \Gamma} \\
\leq C\left\{\left(h^{\min \{\delta, r+1\}}+h^{\min \{\delta, m\}}\right)\|u\|_{1+\delta, \Omega}+\widetilde{h}^{\min \{t-1 / 2, \widetilde{k}\}}\|\lambda\|_{t, \Gamma}\right. \\
\left.+\widehat{h}^{\min \{s, \widehat{k}+1\}-1 / 2}\|\varphi\|_{s, \Gamma}+\widehat{h}^{\min \{\widehat{t}, \widehat{k}\}+1 / 2}\|\gamma\|_{\widehat{t}, \Gamma}\right\},
\end{array}
$$

for all $t>1 / 2, s>1 / 2$, and $\widehat{t}>-1 / 2$.

Proof. If follows straightforward from Theorem 4.1, Lemmas 4.3, 4.4, 4.5, and the approximation properties $\left(\operatorname{AP}\left(Y_{\widehat{h}}^{0}\right)\right)$ and $\left(\operatorname{AP}\left(Z_{\widehat{h}}^{0}\right)\right)$.

As announced in the Introduction, here we observe that the estimate (4.16) is optimal with respect to the meshsizes $h$ and $\widehat{h}$, and suboptimal with respect to $\widetilde{h}$. However, because of the regularity of the exact solution $u$ in the exterior of the support of $f$, the above can be circumvented by assuming $\lambda$ sufficiently regular, whence the optimal rate of convergence is recovered. In particular, we can establish the following corollary.

Theorem 4.3. There exists $C>0$, independent of the meshsizes, such that

$$
\begin{aligned}
& \left\|u-u_{h}\right\|_{h}+\left|\lambda-\lambda_{\widetilde{h}}\right|_{\widehat{h}}+\left\|\sigma-\sigma_{h}\right\|_{0, \Omega}+\left\|\varphi-\varphi_{\widehat{h}}\right\|_{1 / 2, \Gamma}+\left\|\gamma-\gamma_{\widehat{h}}\right\|_{-1 / 2, \Gamma} \\
& \leq C\left\{\left(h^{\min \{\delta, m\}}+\widehat{h}^{\min \{\delta, \widehat{k}+1 / 2\}}\right)\|u\|_{1+\delta, \Omega}+\widetilde{h}^{\min \{1, \widetilde{k}\}}\|\lambda\|_{3 / 2, \Gamma}\right\} .
\end{aligned}
$$

Proof. It suffices to take $s=1 / 2+\delta, \widehat{t}=-1 / 2+\delta$, and $t=3 / 2$. Then, we observe that $h^{\min \{\delta, r+1\}} \leq h^{\min \{\delta, m\}}$ since $r+1 \geq m$, and use that $\|\varphi\|_{1 / 2+\delta, \Gamma}+$ $\|\gamma\|_{-1 / 2+\delta, \Gamma} \leq C\|u\|_{1+\delta, \Omega}$.

It is also clear from (4.16) and (4.17) that $m, \widehat{k}$, and $\widetilde{k}$ must all be $\geq 1$.

We end this paper by remarking that further developments, including nonlinear boundary value problems, a posteriori error analysis, adaptivity, and corresponding numerical experiments, will be reported in forthcoming works.

\section{REFERENCES}

[1] D.N. ARNOLD: Interior penalty finite element method with discontinuous elements. SIAM Journal on Numerical Analysis, vol. 19, 4, pp. 742-760 (1982). MR.0664882 (83f:65173)

[2] D.N. Arnold, F. Brezzi, B. Cockburn and L.D. Marini: Unified analysis of discontinuous Galerkin methods for elliptic problems. SIAM Journal on Numerical Analysis, vol. 39, 5, pp. 1749-1779 (2001). MR1885715 (2002k:65183)

[3] R. Becker, P. HANSBO AND M.G. LARSON: Energy norm a posteriori error estimation for discontinuous Galerkin methods. Computer Methods in Applied Mechanics and Engineering, vol. 192, pp. 723-733 (2003). MR1952357 (2003k:65135)

[4] R. Bustinza, B. Cockburn and G.N. Gatica: An a posteriori error estimate for the local discontinuous Galerkin method applied to linear and nonlinear diffusion problems. Journal of Scientific Computing, vol. 22, 1, pp. 147-185 (2005). MR2142193 
[5] R. Bustinza and G.N. Gatica: A local discontinuous Galerkin method for nonlinear diffusion problems with mixed boundary conditions. SIAM Journal on Scientific Computing, vol. 26, 1, pp. 152-177 (2004). MR2114338 (2005k:65201)

[6] R. Bustinza and G.N. Gatica: A mixed local discontinuous Galerkin method for a class of nonlinear problems in fluid mechanics. Journal of Computational Physics, vol. 207, 2, pp. 427-456 (2005). MR2144625 (2006a:76069)

[7] C. Carstensen and E.P. Stephan: Adaptive coupling of boundary elements and finite elements. Mathematical Modelling and Numerical Analysis, vol. 29, pp. 779-817 (1995). MR.1364401 (97e:65134)

[8] P. Castillo, B. Cockburn, I. Perugia, and D. Schötzau: An a priori error analysis of the local discontinuous Galerkin method for elliptic problems. SIAM Journal on Numerical Analysis, vol. 38, 5, pp. 1676-1706 (2000). MR1813251(2002k:65175)

[9] P. Ciarlet: The Finite Element Method for Elliptic Problems. North-Holland, Amsterdam (1978). MR0520174 (58:25001)

[10] B. CockBuRn AND C. DAWSON: Some extensions of the local discontinuous Galerkin method for convection-diffusion equations in multidimensions. In Proceedings of the 10th Conference on the Mathematics of Finite Elements and Applications, edited by J. Whiteman, Elsevier, pp. 225-238 (2000). MR1801979 (2001j:65142)

[11] B. Cockburn And C.W. Shu: The local discontinuous Galerkin finite element method for convection-diffusion systems. SIAM Journal on Numerical Analysis, vol. 35, 6, pp. 2440-2463 (1998). MR1655854 (99j:65163)

[12] B. CockBURN AND C. DAWSON: Approximation of the velocity by coupling discontinuous Galerkin and mixed finite element methods for flow problems. Locally conservative numerical methods for flow in porous media. Computational Geosciences, vol. 6, 3-4, pp. 505-522 (2002). MR.1956028 (2004b:65179)

[13] M. CostABEL: Boundary integral operators on Lipschitz domains: elementary results. SIAM Journal on Mathematical Analysis, vol. 19, 3, pp. 613-626 (1988). MR0937473 (89h:35090)

[14] C. Dawson And J. Proft: Coupling of continuous and discontinuous Galerkin methods for transport problems. Computer Methods in Applied Mechanics and Engineering, vol. 191, 29-30, pp. 3213-3231 (2002). MR.1908108 (2003d:65090)

[15] C. Dawson And J. Proft: Discontinuous and coupled continuous/discontinuous Galerkin methods for the shallow water equations. Computer Methods in Applied Mechanics and Engineering, vol. 191, 41-42, pp. 4721-4746 (2002). MR1929628 (2003j:76074)

[16] C. Dawson And J. Proft: Coupled discontinuous and continuous Galerkin finite element methods for the depth-integrated shallow water equations. Computer Methods in Applied Mechanics and Engineering, vol. 193, 3-5, pp. 289-318 (2004). MR2031230 (2004k:65170)

[17] G.P. Galdi: An Introduction to the Mathematical Theory of the Navier-Stokes Equations. I: Linearised Steady Problems. Springer-Verlag, 1994. MR.1284205 (95i:35216a)

[18] G.N. Gatica And N. Heuer: A dual-dual formulation for the coupling of mixed-FEM and BEM in hyperelasticity. SIAM Journal on Numerical Analysis, vol. 38, 2, pp. 380-400 (2000). MR1770054 (2001e:65193)

[19] G.N. Gatica AND G.C. HsiaO: Boundary-Field Equation Methods for a Class of Nonlinear Problems. Pitman Research Notes in Mathematics Series, vol. 331, Longman, 1995. MR:1379331 (97k:65269)

[20] G.N. GatiCa AND F.J. SAYAS: A note on the local approximation properties of piecewise polynomials with applications to LDG methods. Complex Variables and Elliptic Equations, vol. 51, 2, pp. 109-117 (2006)

[21] R. HiptMAiR: Coupling of finite elements and boundary elements in electromagnetic scattering. SIAM Journal on Numerical Analysis, vol. 41, 3, pp. 919-944 (2003). MR2005188 (2004i:65121)

[22] P. Houston, J. Robson and E. Süli: Discontinuous Galerkin finite element approximation of quasilinear elliptic boundary value problems I: the scalar case. IMA J. Numer. Anal. vol. 25, 4, pp. 726-749 (2005). MR2170521

[23] A. Marquez, S. Meddahi and V. Selgas: A new BEM-FEM coupling strategy for twodimensional fluid-solid interaction problems. Journal of Computational Physics, vol. 199, 1, pp. 205-220 (2004). MR2081003 (2005b:74041) 
[24] S. Meddahi and F.-J. Sayas: A fully discrete BEM-FEM for the exterior Stokes problem in the plane. SIAM Journal on Numerical Analysis, vol. 37, 6, pp. 2082-2102 (2000). MR1766860 (2001e:65185)

[25] S. Meddahi And V. Selgas: A mixed-FEM and BEM coupling for a three-dimensional eddy current problem. Mathematical Modelling and Numerical Analysis, vol. 37, 2, pp. 291-318 (2003). MR.1991202 (2004f:78007)

[26] S. Meddahi, J. Valdés, O. Menéndez, And P. PÉrez: On the coupling of boundary integral and mixed finite element methods. Journal of Computational and Applied Mathematics, vol. 69, pp. 113-124 (1996). MR.1391614 (97e:65137)

[27] I. Perugia And D. SснӧтzAU: On the coupling of local discontinuous Galerkin and conforming finite element methods. Journal of Scientific Computing, vol. 16, 4, pp. 411-433 (2001). MR.1881853 (2002k:65194)

[28] I. PeRugia AND D. SchötZau: An hp-analysis of the local discontinuous Galerkin method for diffusion problems. Journal of Scientific Computing, vol. 17, 1-4, pp. 561-571 (2002). MR.1910752

[29] B. Riviere And M.F. WheEler: A posteriori error estimates and mesh adaptation strategy for discontinuous Galerkin methods applied to diffusion problems. Preprint 00-10, TICAM, University of Texas at Austin, USA (2000).

[30] F.-J. SAYAS: A nodal coupling of finite and boundary elements. Numerical Methods for Partial Differential Equations, vol. 19, 5, pp. 555-570 (2003). MR.1996220 (2004g:65160)

[31] S. Sun, B. Riviere And M.F. Wheeler: A combined mixed finite element and discontinuous Galerkin method for miscible displacement problem in porous media. Recent progress in computational and applied PDEs (Zhangjiajie, 2001), pp. 323-351, Kluwer/Plenum, New York, 2002. MR2039576 (2005b:65110)

Departamento de Ingeniería Matemática, Universidad de Concepción, Casilla 160-C, Concepción, Chile

E-mail address: ggatica@ing-mat.udec.cl

Departamento de Matemática Aplicada, Universidad de Zaragoza, Centro Politécnico Superior, María de Luna, 3 - 50018 Zaragoza, Spain

E-mail address: jsayas@unizar.es 